\title{
The Three-Dimensional Structure of Breaking Rossby Waves in the Polar Wintertime Stratosphere
}

\author{
L. M. Polvani \\ Department of Applied Physics and Applied Mathematics, Columbia University, New York, New York \\ R. SARAVANAN \\ National Center for Atmospheric Research, Boulder, Colorado
}

(Manuscript received 13 May 1999, in final form 7 December 1999)

ABSTRACT

\begin{abstract}
The three-dimensional nature of breaking Rossby waves in the polar wintertime stratosphere is studied using an idealized global primitive equation model. The model is initialized with a well-formed polar vortex, characterized by a latitudinal band of steep potential vorticity (PV) gradients. Planetary-scale Rossby waves are generated by varying the topography of the bottom boundary, corresponding to undulations of the tropopause. Such topographically forced Rossby waves then propagate up the edge of the vortex, and their amplification with height leads to irreversible wave breaking.

These numerical experiments highlight several nonlinear aspects of stratospheric dynamics that are beyond the reach of both isentropic two-dimensional models and fully realistic GCM simulations. They also show that the polar vortex is contorted by the breaking Rossby waves in a surprisingly wide range of shapes.

With zonal wavenumber-1 forcing, wave breaking usually initiates as a deep helical tongue of PV that is extruded from the polar vortex. This tongue is often observed to roll up into deep isolated columns, which, in turn, may be stretched and tilted by horizontal and vertical shears. The wave amplitude directly controls the depth of the wave breaking region and the amount of vortex erosion. At large forcing amplitudes, the wave breaking in the middle/lower portions of the vortex destroys the PV gradients essential for vertical propagation, thus shielding the top of the vortex from further wave breaking.

The initial vertical structure of the polar vortex is shown to play an important role in determining the characteristics of the wave breaking. Perhaps surprisingly, initially steeper PV gradients allow for stronger vertical wave propagation and thus lead to stronger erosion. Vertical wind shear has the notable effect of tilting and stretching PV structures, and thus dramatically accelerating the downscale stirring. An initial decrease in vortex area with increasing height (i.e., a conical shape) leads to focusing of wave activity, which amplifies the wave breaking. This effect provides a geometric interpretation of the "preconditioning" that often precedes a stratospheric sudden warming event. The implications for stratospheric dynamics of these and other threedimensional vortex properties are discussed.
\end{abstract}

\section{Introduction}

The most prominent feature of the wintertime stratosphere is a band of strong circumpolar westerly winds whose latitudinal extent defines the edge of the polar night vortex. The strong latitudinal gradients of potential vorticity (PV) found at the edge of the vortex are believed to isolate polar air inside the vortex from air at lower latitudes, so much so that the polar vortex has been referred to as a "containment vessel" (Juckes and McIntyre 1987; see also Randel 1993). Within the vortex, extremely low temperatures allow for the formation

Corresponding author address: Dr. Lorenzo Polvani, Dept. of Applied Physics and Applied Mathematics, Columbia University, New York, NY 10027.

E-mail: polvani@columbia.edu of polar stratospheric clouds on which chemical reactions leading to the eventual destruction of ozone are known to occur. Understanding how polar, ozone-depleted air is transported to other parts of the atmosphere is thus a question of major importance (Schoeberl and Hartman 1991; McIntyre 1991).

It is now widely accepted that the principal mechanism for mixing polar air into the midlatitudes is the breaking of planetary-scale Rossby waves (McIntyre and Palmer 1983, 1984). These waves propagate upward from the troposphere and upon breaking distort the otherwise circular vortex, literally tearing away polar air from the edges of the vortex and stirring it into the midlatitudes. Both satellite measurements of long-lived tracers (Leovy et al. 1985) and in situ aircraft measurements combined with trajectory-following techniques (Plumb et al. 1994; Waugh et al. 1994) have 
shown how such vortex stripping by the Rossby waves erodes the polar vortex, and generates a "surf zone" where polar air is kinematically stirred into extremely finescaled filaments. Moreover, multilevel contour advection studies using analyzed winds (Schoeberl and Newman 1995; Mariotti et al. 1997) have shown that these filaments are, in fact, sheets of anomalous air.

On the theoretical side much progress has been made as well. Since the pioneering work of Juckes and McIntyre (1987), several idealized two-dimensional studies have shed much light on how wave breaking proceeds on near-horizontal (isentropic) surfaces (Juckes 1989; Salby et al. 1990a-c; Polvani and Plumb 1992; Waugh 1993; Yoden and Ishioka 1993; Norton 1994; Polvani et al. 1995). By their very nature, however, these one-layer models have little insight to offer on the vertical structure of the breaking Rossby waves. On the other hand, many realistic three-dimensional models of the stratosphere have succeeded in reproducing observed features of the circulation (O'Neill and Pope 1988; Cariolle et al. 1993; Jackson and Gray 1994; Hamilton et al. 1995; Boville 1995). Given their inevitable complexity, however, such realistic models have been unable to provide a clear picture of individual wave-breaking events. The present work is aimed at filling this gap.

Using an idealized but fully three-dimensional primitive-equation model we are able to capture in detail how the entire three-dimensional shape of the polar vortex is contorted by breaking Rossby waves. On the one hand, the relative simplicity of our model allows us to perform a fairly large number of simulations and thus to determine the influence of key physical parameters on the dynamical evolution of the breaking waves. On the other, the full three-dimensionality of our model reveals that breaking Rossby waves can twist the polar vortex into surprisingly complex and varied shapes. Before presenting our own results, we briefly review three recent papers that have started to address specifically the vertical structure of breaking Rossby waves.

Haynes (1990) has presented two idealized simulations using a forty-level hemispherical primitive-equation model forced at the bottom level. These simulations show that the stripping of vortex filaments and the formation of a surf zone-key features of stratospheric wave breaking demonstrated by Juckes and McIntyre (1987) with a one-layer model-persist when the full vertical structure of the vortex is accounted for.

Dritschel and Saravanan (1994, hereafter DS94), on the other hand, have used multilayer quasigeostrophic contour dynamics to explore how wave breaking on an initially cylindrical vortex varies with forcing amplitude. Using a topographic forcing at the bottom of the vortex to generate upwardly propagating Rossby waves, they discovered two distinct wave-breaking regimes. For weak forcing amplitude the main breaking region was found near the top of the vortex, while for large forcing amplitude it was found at the bottom of the vortex (i.e., near the forcing region). For this reason, they named the first regime "remote" breaking, and the second "local" breaking. In the second regime, the horizontal PV gradients near the bottom of the vortex were destroyed by the wave breaking, thus "shielding" the top of the vortex from vertically propagating waves.

Waugh and Dritschel (1999, hereafter WD99), using the same contour dynamics model, have explored the further question of how the wave breaking depends on the shape of the vortex (i.e., whether it is cylindrical or conical) and on the vertical wind shear associated with the vortex. They have found that a vertical increase of either vortex area or zonal mean winds reduces the upward wave propagation and eliminates the remote breaking at weak forcing amplitudes reported by DS94, so that only local breaking is observed. However, when the two effects are combined (i.e., for conical and vertically sheared vortices), both local and remote breaking are found. One of the purposes of the present study is to what extent the three-dimensional features of Rossby wave breaking in contour dynamics models are also present in a more realistic, yet still highly idealized, primitive equation model.

Our paper proceeds as follows. In section 2 we discuss the physical and numerical characteristics of our model, we explain how polar vortices with varying shapes, vertical wind shears, and PV gradients are constructed to provide a variety of initial conditions, and we describe how vertically propagating Rossby waves are forced at the lower boundary. In section 3 we study the threedimensional evolution of breaking Rossby waves on initially barotropic vortices (i.e., such that the initial flow is independent of the vertical coordinate), and we explore how the wave breaking varies as the forcing amplitude and the PV gradient of the initial vortex are varied. In section 4 we focus on initially baroclinic vortices, and study how the initial conical shape and vertical wind shear affect the wave-breaking process. While, in all the above, we use zonal wavenumber-1 forcing, associated with the typical "comma"-shaped on isentropic surfaces, in section 5 we take a look at wavenumber- 2 forcing, associated with a breakup of the vortex into two distinct parts, a behavior typical of so-called sudden-warming events. A discussion concludes the paper.

\section{The model}

In the spirit of geophysical fluid dynamics, we have chosen a physical model with minimal complexity yet sufficient realism. Since the principal focus of this study is the vertical structure of Rossby wave breaking in the stratosphere, we have opted to avoid the complications of implementing a faithful representation of the troposphere. Our model is thus comprised of a single fully spherical density-stratified shell of fluid; it is meant to represent the stratosphere alone. The bottom of our model, which is a material surface, plays the role of the 
tropopause. For this reason, the reference surface pressure in our model is $100 \mathrm{hPa}$.

The fluid in our model obeys the primitive equations in spherical coordinates. Since our objective is to study the vortex dynamics associated with Rossby wavebreaking events, and since such events are relatively short (typically of the order of 10-20 days), we have excluded nearly all diffusive, orographic, radiative, moist, and chemical effects from our model. Our aim is to solve nearly adiabatic primitive equations.

In fact, the numerical solutions presented in this paper were obtained with an idealized version of NCAR's Community Climate Model 2 (Hack et al. 1993), from which we removed everything (e.g., cloud parameterizations, ozone data, surface fluxes, etc.) other than the adiabatic primitive equations, that is, the dynamical core. This dynamical core solves the primitive equations with a semi-implicit leapfrog time stepping stabilized by a Robert-Asselin filter, and a spatial discretization that is pseudospectral in the horizontal direction and finite difference in the vertical. Unless otherwise specified, all the results presented in this paper were obtained with a horizontal spectral truncation of T42, for which the collocation grid has a $2.8^{\circ}$ spacing. This resolution is adequate for the purposes of the present work (as a check, several runs were repeated at the higher resolution T85). As for vertical resolution, we have used 48 layers to span $60 \mathrm{~km}$ in height from the nominal tropopause to the top of our model.

To perform our numerical experiments, we have made only three additions to the bare dynamical core: a topographic surface forcing to efficiently generate upwardly propagating Rossby waves, a sponge layer to prevent unrealistic wave reflection from the top of the model, and a numerical viscosity to effectively dissipate the small scales produced during the wave-breaking events. We discuss each one in detail.

The surface forcing was introduced by taking advantage of the fact that the vertical coordinate in our model is a hybrid of pressure and $\sigma$ (Simmons and Strufing 1981). It varies from pure $\sigma$ at the surface to pure pressure higher in the model with a hyperbolic tangent profile, centered at $1.5 H_{0}$ and of width $H_{0} / 2$, the reference scale height in our model being $H_{0}=7 \mathrm{~km}$. This means that $18 \mathrm{~km}$ above the surface, the vertical coordinate is pure pressure, as one would desire for a stratospheric model. However, the significance of a hybrid vertical coordinate choice rests in that it allows the bottom level of our model to behave as a material surface, unlike other mechanistic models (e.g., Boville 1995). While the actual tropopause may not in itself act as a material surface, our choice has the benefit of at least being a physically realizable system (if only laboratory experiments with stratified rotating spherical fluid shells could be easily performed!).

To gather some ideas as to what spatial and temporal variations may be used to specify the topographic forcing at the bottom of our model, we have thought it useful to look at some actual data. It should be made perfectly clear that we are not, in this study, trying to simulate any particular stratospheric event. We simply wish to use observations to guide our choices, so that our idealized model be at least partially relevant to the real world.

As a representative case of a strong zonal breaking event leading to the familiar "comma" shape, we have selected the celebrated winter of 1979 event analyzed by McIntyre and Palmer (1984). In Fig. 1a we show the eddy geopotential at $100 \mathrm{hPa}$ on 26 January 1979 from the National Centers for Environmental Prediction-National Center for Atmospheric Research (NCEP-NCAR) global reanalysis data set (Kalnay et al. 1996). The dominant zonal wavenumber $m=1$ is readily apparent. We have also extracted the time-latitude evolution of the $m$ $=1$ component of the geopotential at $100 \mathrm{hPa}$ for the period 10 January-18 February 1979, and plotted it in Fig. $1 b$.

For $m=2$ forcing, we have looked at the classic sudden warming event discussed in Andrews et al. (1987, 263-264). The full eddy geopotential at $100 \mathrm{hPa}$ on 21 February 1979, the day when the $m=2$ amplitude is maximal, shows that the field is almost totally dominated by the $m=2$ mode (Fig. $2 \mathrm{a}$ ). The $m=2$ component of the geopotential at $100 \mathrm{hPa}$ from NCEP for the period 10 February-12 March 1979 is plotted in Fig. $2 b$.

From these data, the key features of the geopotential perturbations in the uppermost troposphere associated with strong wave-breaking events are readily apparent. First, the latitudinal extent is well confined to a band extending roughly from $40^{\circ} \mathrm{N}$ to $80^{\circ} \mathrm{N}$. Second the longitudinal component is clearly dominated, at least in the above two examples, by a single zonal wavenumber. Third, the time variation appears to be in the form of a pulse, with a width of the order of 10-20 days.

These observations provide useful guidelines for constructing a simple analytic form for the forcing function that we use to generate upwardly propagating Rossby waves in our model. This is done by specifying a bottom topography (i.e., the surface geopotential $\Phi_{S}$ ), as a function of space and time, according to the expression

$$
\Phi_{S}(\lambda, \phi, t)=\Phi_{A} \cos (m \lambda) F(\phi) T(t),
$$

where $\Phi_{A}$ is the forcing amplitude (in meters), $\lambda$ the longitude, and $\phi$ the latitude. For the longitudinal dependence, we have considered pure $m=1$ and $m=2$ zonal wavenumbers. The latitudinal extent of the forcing, represented by $F(\phi)$, is confined between two latitudes $\phi_{1}$ and $\phi_{2}$, and has the functional form

$$
F(\phi)=\left[\sin \left(\pi \frac{\phi-\phi_{1}}{\phi_{2}-\phi_{1}}\right)\right]^{2}
$$

for $\phi_{1}<\phi<\phi_{2}$, and $F(\phi)=0$ otherwise. With the choices $\phi_{1}=40^{\circ} \mathrm{N}$ and $\phi_{2}=80^{\circ} \mathrm{N}$, this forcing profile, 

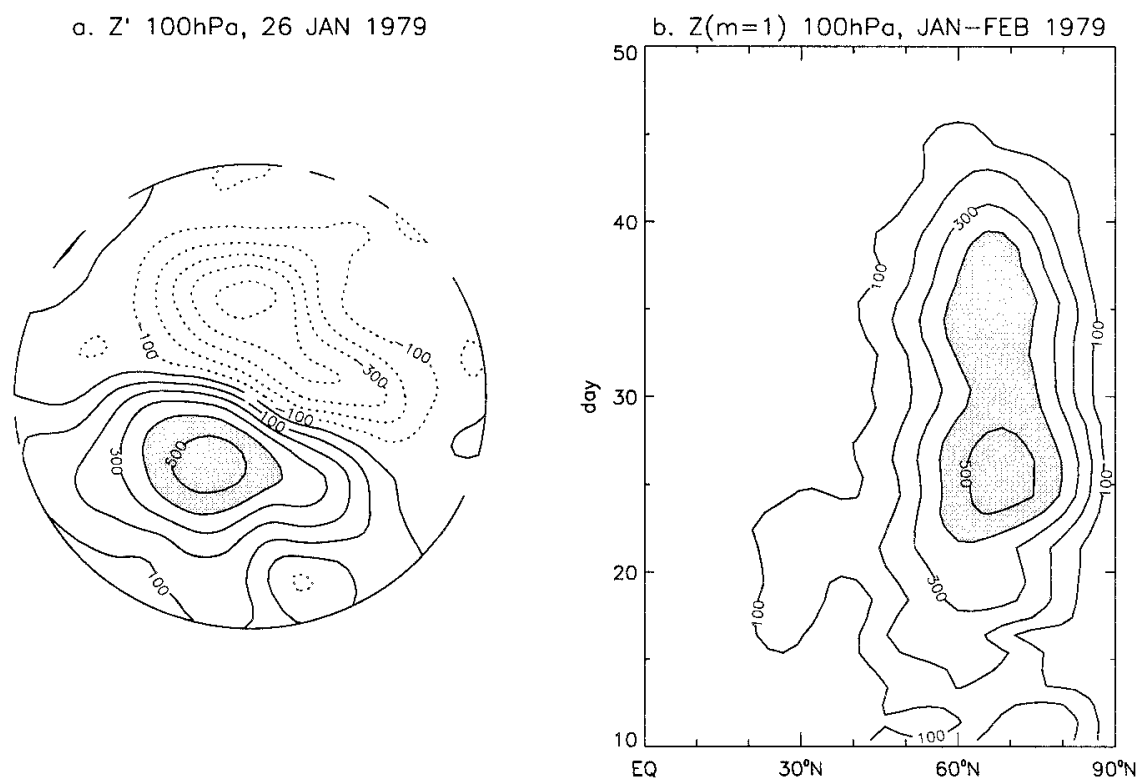

FIG. 1. The geopotential field corresponding to the event analyzed by McIntyre and Palmer (1984). (a) The observed eddy geopotential height at $100 \mathrm{hPa}$ on 26 Jan 1979. (b) Time evolution of the $m=1$ component of the eddy geopotential for the period 10 Jan-18 Feb 1979 .

illustrated in Fig. 3a, peaks at $60^{\circ} \mathrm{N}$. Finally, the temporal evolution of the forcing is given by

$$
T(t)=\left[1-e^{-\left(t-t_{\mathrm{on}}\right) / \tau}\right]\left[1-e^{\left(t-t_{\mathrm{off}}\right) / \tau}\right]
$$

for $t_{\mathrm{on}}<t<t_{\mathrm{off}}$, and $T(t)=0$ otherwise. We have investigated two types of time dependence. The first type, obtained by choosing $t_{\mathrm{on}}=0, t_{\mathrm{off}}=12$ days, and $\tau=3$ days, will be referred to as "pulsed" forcing; it is illustrated by the solid line in Fig. 3b, and is similar in character to the observations shown in Figs. 1, 2. The second type, referred to as "steady" forcing, is shown by the dashed line in Fig. 3b, and corresponds to $t_{\mathrm{on}}=0, t_{\mathrm{off}}=\infty$ days, and $\tau=3$ days. The reason for considering such steady forcing is that it has been used in almost all previous idealized studies of Rossby wave breaking in the stratosphere. o. Z' $100 \mathrm{hPa}, 21 \mathrm{FEB} 1979$

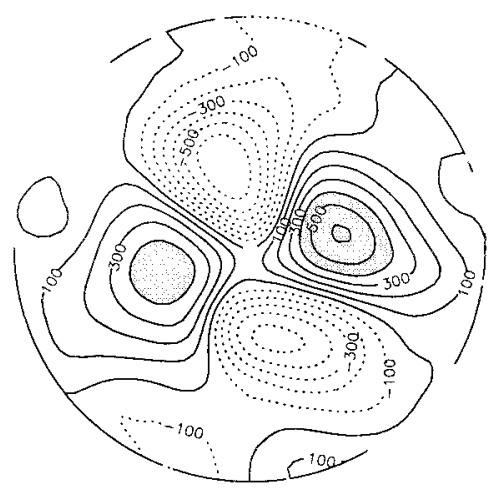

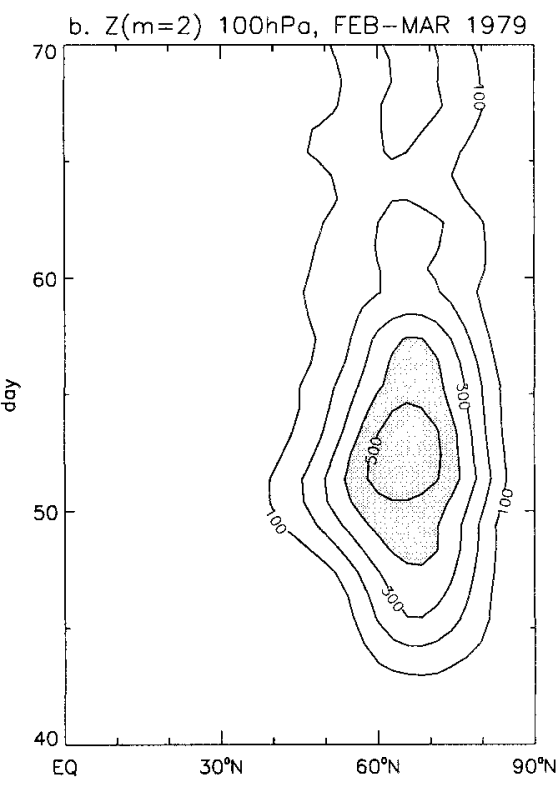

FIG. 2. The geopotential field corresponding to the "sudden warming" event described in Andrews et al. (1987). (a) The observed eddy geopotential height at $100 \mathrm{hPa}$ on $21 \mathrm{Feb} 1979$. (b) Time evolution of the $m=2$ component of the eddy geopotential for the period 10 Feb-12 Mar 1979. 

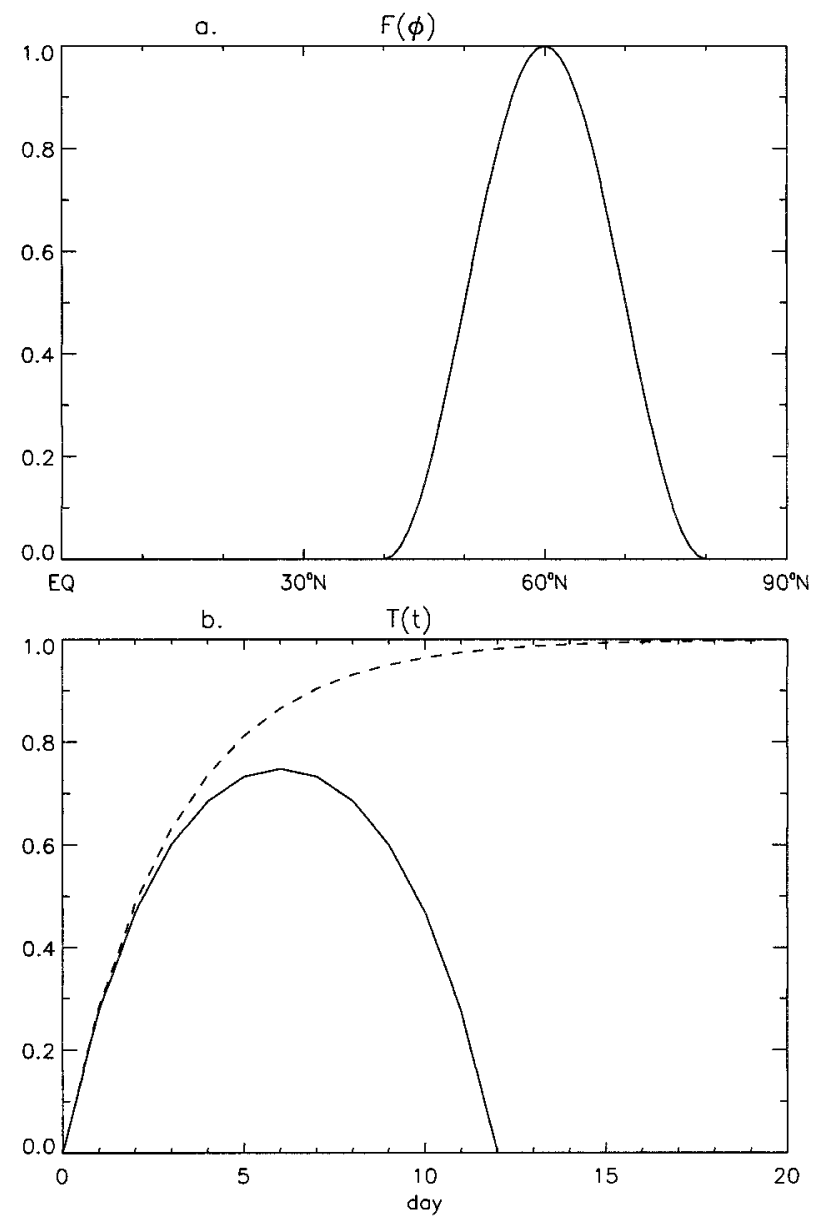

FIG. 3. The forcing function used in the numerical experiments presented in this paper. (a) The latitudinal dependence $F(\phi)$, and (b) the temporal dependence $T(t)$. In (b), the solid line is referred to in the text as "pulsed" forcing, and the dotted line as "steady" forcing.

Finally, we have chosen the amplitude $\Phi_{A}$ in Eq. (1) by plotting the geopotential height on an isentropic surface in the lower stratosphere. On the $462-\mathrm{K}$ potential temperature surface (corresponding to a pressure of 100 $\mathrm{hPa}$ where the bottom of our model is located), plots of geopotential height for the two events shown in Figs. 1 and 2 show maxima around $1800 \mathrm{~m}$. Hence, we decided to explore in our numerical experiments the range $200 \mathrm{~m}<\Phi_{A}<2000 \mathrm{~m}$, and we have used the value $\Phi_{A}=800 \mathrm{~m}$ for a "typical" forcing amplitude.

The second ingredient we have added to the bare dynamical core is a sponge layer near the top of our model; it is needed to prevent the spurious reflection of Rossby waves from the top of the model. We have implemented the sponge layer by adding a Rayleigh damping term that acts, with an $e$-folding scale of 1 day, on the eddy component of the velocity field. There is also a Newtonian damping term in the sponge layer, with the same timescale, that acts on the thermal anomalies. Vertically, the sponge layer has a hyperbolic tangent profile, centered $52.5 \mathrm{~km}$ above the surface and with a width of $5 \mathrm{~km}$; in practice, this means that the top 12 layers of our model are affected by this sponge. In what follows, we show only the lowest $45 \mathrm{~km}$, where the flow is not directly affected by the presence of a sponge layer above.

The third addition to the dynamical core is hyperdiffusion. A term proportional to $\nabla^{8}$, instead of CCM2's original $\nabla^{4}$, was added in the vorticity, divergence, and temperature equations. Such hyperdiffusion is customary in spectral models, and is needed to control the enstrophy cascade to small scales. We have used a hyperdiffusion coefficient such that the smallest resolved wavenumber is diffused with an $e$-folding time of half a day; a few runs were repeated with an $e$-folding time of one-quarter of a day, and no noticeable differences appeared in the results.

The last point that needs discussion before we present our results, is the initialization of the polar vortex. Since the main objective of this study is to understand the vertical structure of Rossby waves as they propagate up the polar vortex edge and eventually break, we need to initialize the flow with a well-formed polar vortex already present. This is not difficult to do, but the details are somewhat tedious, and are thus relegated to the appendix. Here we simply sketch the basic procedure.

Briefly, a number of parameters are chosen to set up a desired zonally symmetric PV profile. This is then inverted and balanced so as to obtain initial velocities, temperature, and surface pressure with which the model is initialized. The key parameters that control the initial PV distribution are $U_{v b}$ and $U_{v t}$, the zonal wind at the vortex edge (at the bottom and top of the vortex, respectively); $\phi_{v b}$ and $\phi_{v t}$, the latitudinal location of the vortex edge (also at the bottom and top); and $\Delta \phi$, the thickness of the vortex edge. These parameters (and a few others, cf. the appendix) allow us to control the vertical wind shear at the vortex edge, the geometrical shape of the vortex (cylindrical or conical), the steepness of the PV gradients at the vortex edge, the latitudinal location of the zero-wind line, and the strength of the easterlies at the equator. In other words, they allow us to construct idealized yet realistic initial flows. Table 1 summarizes the five initial conditions we have considered in this study. Our reference case is labeled BAROT (barotropic) and corresponds to an initially cylindrical vortex with no vertical wind shear, such as the one considered by DS94. This simplest case is the one we consider first.

\section{Barotropic vortex}

For clarity, we start by considering an initially isothermal vortex, that is, such that the initial temperature is spatially uniform. Even in this simplest case, however, it is well known that the exponential dependence of density on height implies an exponential increase of the Ertel PV with height (Hoskins et al. 1985). As pointed out by Lait (1994), "this dependence makes it difficult 
TABLE 1. Vortex parameters for the different initial conditions used in this paper.

\begin{tabular}{|c|c|c|c|c|c|c|}
\hline \multirow[b]{2}{*}{ Name } & $U_{v \mathrm{~b}}$ & $U_{v \mathrm{t}}$ & $\phi_{v b}$ & $\boldsymbol{\phi}_{v \mathrm{t}}$ & \multirow{2}{*}{$\frac{\Delta_{\phi}}{{ }^{\circ} \text { lat }}$} & \multirow[b]{2}{*}{ Description } \\
\hline & \multicolumn{2}{|c|}{$\mathrm{m} \mathrm{s}^{-1}$} & \multicolumn{2}{|c|}{${ }^{\circ} \mathrm{N}$} & & \\
\hline BAROT & 60 & 60 & 60 & 60 & 6 & Cylindrical vortex edge with no vertical wind shear \\
\hline SHEAR & 60 & 120 & 60 & 60 & 6 & Cylindrical vortex edge with vertical wind shear \\
\hline SLOPE & 60 & 60 & 60 & 45 & 6 & Sloping vortex edge with no vertical wind shear \\
\hline SLOSH & 60 & 120 & 60 & 45 & 6 & Sloping vortex edge with vertical wind shear \\
\hline SMOOTH & 60 & 60 & 60 & 60 & 20 & As in BAROT, but for a smoother vortex edge \\
\hline
\end{tabular}

to compare horizontal maps at different potential temperature levels, or to view horizontal variations in a vertical cross section of the atmosphere." For visualizing fully three-dimensional fields, therefore, a scaled dynamical quantity is needed. Following Lait (1994), we define scaled PV (hereafter SPV) as ertel PV divided by a factor of

$$
\left[\frac{g \kappa f}{p_{0} T_{0}^{1 / \kappa}} \theta_{0}^{(\kappa+1) / \kappa}\right]\left(\frac{\theta}{\theta_{0}}\right)^{(\kappa+1) / \kappa},
$$

where $T_{0} \equiv\left(g H_{0}\right) / R$ is the reference temperature, $p_{0}=$ $100 \mathrm{hPa}$ is a reference pressure, and $\theta_{0}$ is the potential temperature at $p_{0}$; all other symbols are standard. With our choice of $H_{0}=7 \mathrm{~km}$, we have $T_{0}=239 \mathrm{~K}$ and $\theta_{0}$ $=461 \mathrm{~K}$. Notice that the quantity in brackets has dimensions of PV; it is simply the PV scale at $\theta_{0}$. Thus $\mathrm{SPV}$, as we have defined it, is an $O(1)$ nondimensional quantity, varying from 0 at the equator to about 1.3 at the pole.

The initial height-latitude distribution of SPV is plotted in Fig. 4a. The barotropic nature of our initial condition is now explicit. As already mentioned, we initialize our model with a flow in which the polar vortex is well formed; this is apparent in the tight meridional gradients of $\mathrm{PV}$ around $60^{\circ} \mathrm{N}$, where the vortex edge is located. The corresponding initial winds are illustrated in Fig. 4b; they are also independent of height, reach a maximum of westerly speed of $60 \mathrm{~m} \mathrm{~s}^{-1}$ at the vortex edge, and are weak and easterly at the equator and in the Southern Hemisphere.

Given such an initial condition, the upward propagation and breaking of topographically forced Rossby waves is illustrated in the top row of Fig. 5, for a pulsed topographic forcing with amplitude $\Phi_{A}=800 \mathrm{~m}$ and zonal wavenumber $m=1$. The figure shows, at selected days, the 0.8 isosurface ${ }^{1}$ of SPV. This value was chosen as representative of the vortex edge (cf. Fig. 4).

Although the forcing is located at the bottom of the model, the amplification of the perturbations with height leads to the formation of the familiar comma shape at the top of the vortex first (day 8). Note that the PV "tongue" has a longitudinally dependent vertical extent; it is deeper closer to the vortex edge, and its tip is the shallowest. Observed wave breaking in the stratosphere exhibits similar features (Schoeberl and Newman 1995). By day 12, the top third of the vortex has been stretched and folded into a vertically aligned annular sheet of PV, which then rolls up into a small number of columnar structures (day 20). Owing to the relatively coarse horizontal grid spacing we are using, very fine structures

\footnotetext{
${ }^{1}$ Before extracting the desired isosurface, the model data was projected from the Gaussian spherical collocation grid onto a rectangular stereographic Cartesian grid centered at the pole and extending equatorward to a latitude of $10^{\circ} \mathrm{N}$.
}
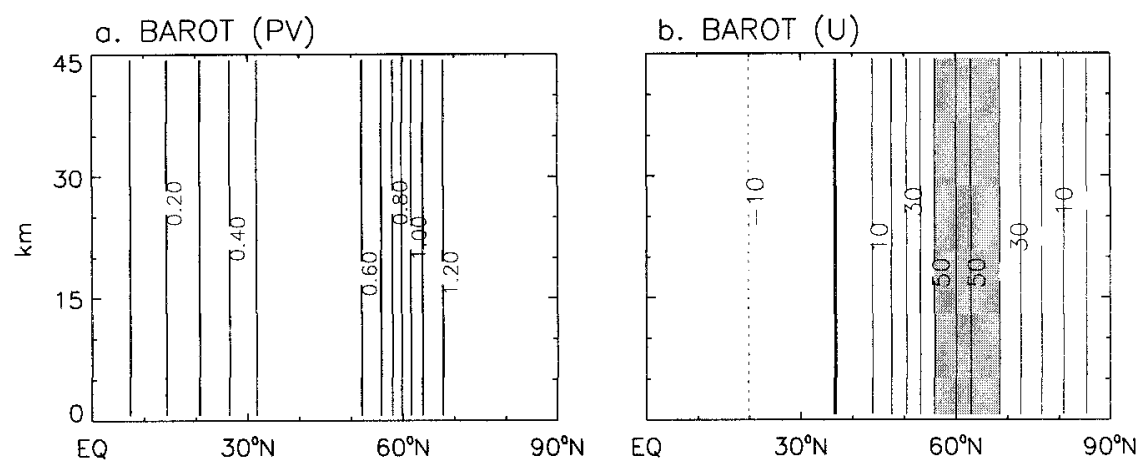

FIG. 4. (a) The scaled potential vorticity (SPV) for the initially barotropic vortex (case BAROT in Table 1). Note the steep gradients centered at $60^{\circ} \mathrm{N}$, indicating the vortex edge. The contour interval is 0.1 (see text for an explanation of the nondimensional quantity SPV). (b) The corresponding zonal winds. The contour interval is $10 \mathrm{~m} \mathrm{~s}^{-1}$; solid lines are westerlies, dotted lines are easterlies, and the thick line is the zero wind line. The shaded area highlights the winds in excess of $50 \mathrm{~m} \mathrm{~s}^{-1}$ (i.e., the vortex edge). 


\begin{tabular}{|l|l|l|l|l|}
\hline day 8 & day 12 & day 16 & day 20 & day 27 \\
\hline & & & \\
\hline
\end{tabular}

FIG. 5. The vortex edge (SPV $=0.8$ isosurface), at selected times, during the evolution and breaking of a topographically forced Rossby wave $\left(\Phi_{A}=800 \mathrm{~m}, m=1\right)$. Top row: pulsed forcing case. Bottom row: steady forcing case. The vortex is originally cylindrical (case BAROT, Fig. 4).

cannot be resolved and are eventually dissipated. Thus, toward the end of the run (day 27), the top third of the vortex has been eroded (and dissipated) away.

In Fig. 6 we show two commonly used diagnostics that quantify this wave-breaking event. The zonal wind (Fig. 6a), longitudinally averaged at $60^{\circ} \mathrm{N}$, shows how the breaking waves deposit easterly momentum in the surf zone, yielding a deceleration of the zonal winds. Similarly, the model temperature over the polar region (Fig. 6b), that is, zonally averaged at $80^{\circ} \mathrm{N}$, shows the characteristic warming associated with the destruction of the vortex.

In the case of steady forcing, the results of a similar computation are shown in the bottom row of Fig. 5. Little difference is apparent at early times. By day 16, however, it is clear that the wave-breaking region is much deeper when the forcing is not turned off; the top half of the vortex is now being distorted into a spiral. This spiral shows a considerable degree of vertical alignment, extending over several scale heights, and forming a nearly vertical sheet of PV. This sheet again rolls up into a number of deep columnar structures, which are clearly seen at day 27 . Similar vortex roll-up structures were noted even in the coarse-grained isentropic PV maps of McIntyre and Palmer (1984). Finally, notice how the planetary waves basically destroy the
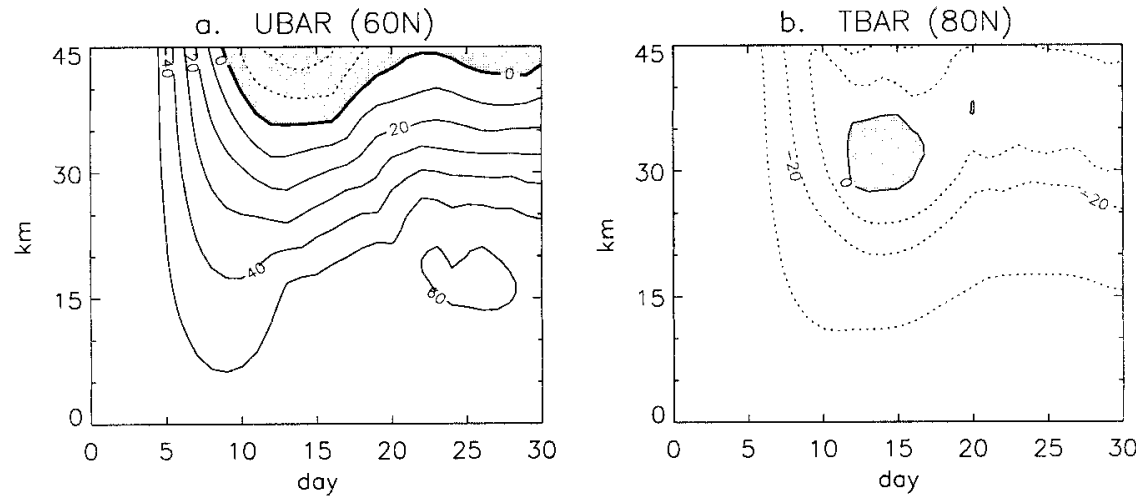

FIG. 6. The time evolution of the zonally averaged (a) zonal winds at $60^{\circ} \mathrm{N}$, and (b) temperature at $80^{\circ} \mathrm{N}$, for the wave-breaking event in Fig. 5, top row. The contour intervals are $10 \mathrm{~m} \mathrm{~s}^{-1}$ in (a) and $10^{\circ} \mathrm{C}$ in (b). Shaded areas correspond to easterlies in (a) and above zero temperatures in (b). 

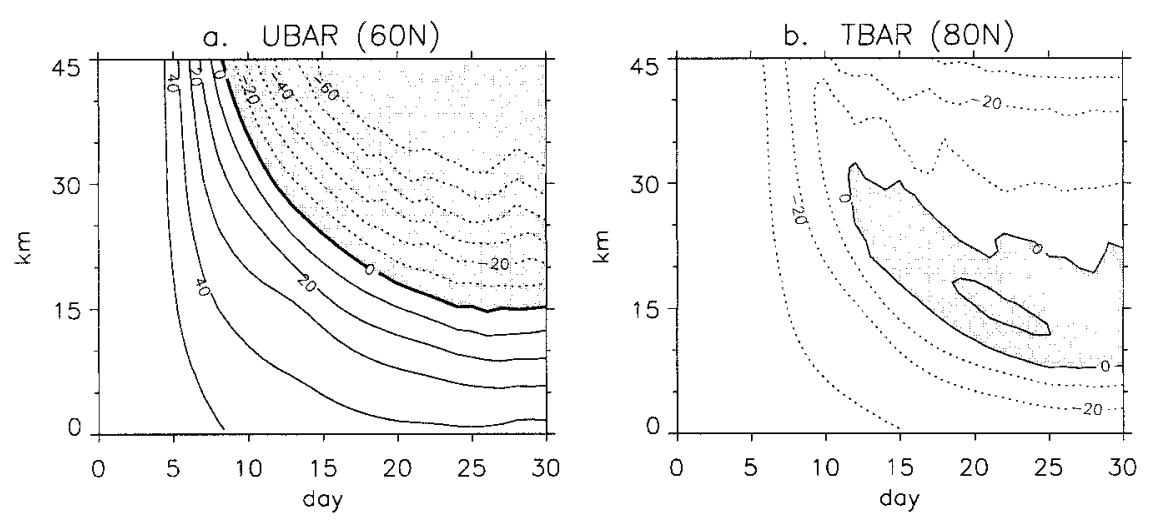

FIG. 7. As in Fig. 6 but for the steady forcing experiment in the bottom row of Fig. 5.

entire vortex when the forcing is steady, whereas in the pulsed forcing experiment only the top third of the vortex is destroyed.

We have found the roll-up of the PV sheets to be a ubiquitous behavior in our numerical experiments, whereas they seem to be somewhat rare in the numerical experiments of DS94 with multilayer contour dynamics. One possible explanation for this discrepancy is that, in our experiments, the PV sheets are formed from the stretching out of the polar vortex itself; hence, once the sheets are stretched out, no polar vortex is present at nearby levels to provide a stabilizing adverse shear (Dritschel and Polvani 1992). In the experiments of DS94, in contrast, the polar vortex itself never really gets destroyed, and may thus provide enough adverse shear to stabilize the PV sheets.

The zonal wind and temperature diagnostics for the steady forcing case (Fig. 7) show how much more the waves disrupt the vortex when the forcing is steady. The easterlies are much stronger (Fig. 7a) and penetrate a lot deeper. Similarly, the warming (Fig. 7b) is found to peak in the lower half of the vortex.

\section{a. Steepness of the PV gradients}

Since the propagation of Rossby waves is made possible by the existence of PV gradients, one might ask how the evolutions we have just described might be affected if the PV gradients were different. To explore this, we have constructed the initial condition SMOOTH (cf. Table 1) shown in Fig. 8a, where the PV gradient at the vortex edge is considerably weaker than that in the previous section (case BAROT, cf. Fig. 4a). Such smoother PV profiles result, for instance, when climatological averages of the polar wintertime stratosphere are taken, owing to the wobbling of the vortex off the pole. Most previous studies of wave breaking in the stratosphere that have used realistic models (e.g., O'Neill and Pope 1988) have employed such smoother initial conditions. We have taken care to initialize the smoother edged case SMOOTH with winds that are as similar as possible to those of case BAROT, as can be seem from in Fig. 8b. In particular, crucial features such as the wind maximum and the zero wind line are located at identical latitudes in both cases.

The evolution of this initially smoother vortex perturbed with a $\Phi_{A}=800 \mathrm{~m}$ pulsed forcing, with zonal wavenumber $m=1$, is shown in the top row of Fig. 9 . This should be contrasted with the evolution in the top row of Fig. 5, in that the two computations are identical except for the initial conditions. Notice the dramatic effect of the smoother PV gradients in suppressing the wave-breaking process. The vortex is left basically intact, except for a tiny filament at the very top. A similar
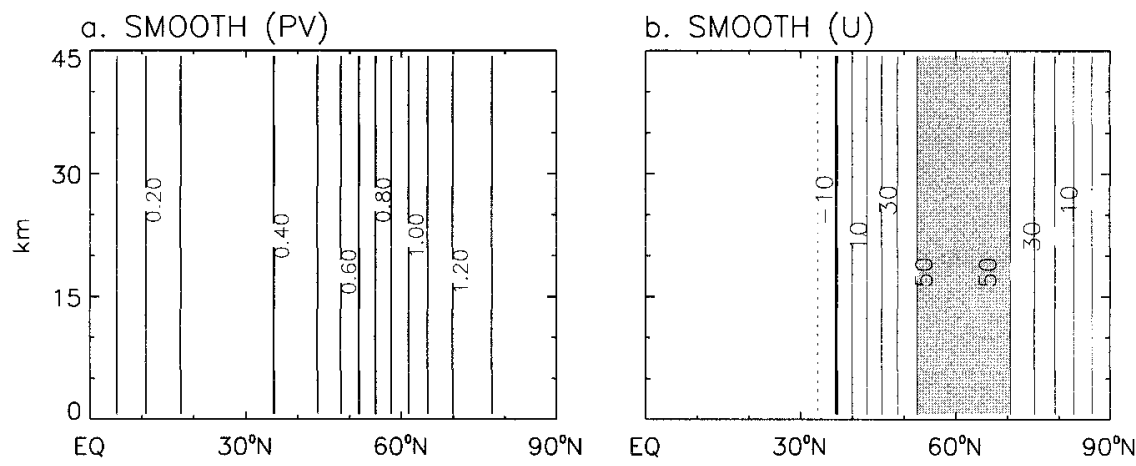

FIG. 8. As in Fig. 4 but for a vortex with smoother initial PV gradients (case SMOOTH in Table 1). 


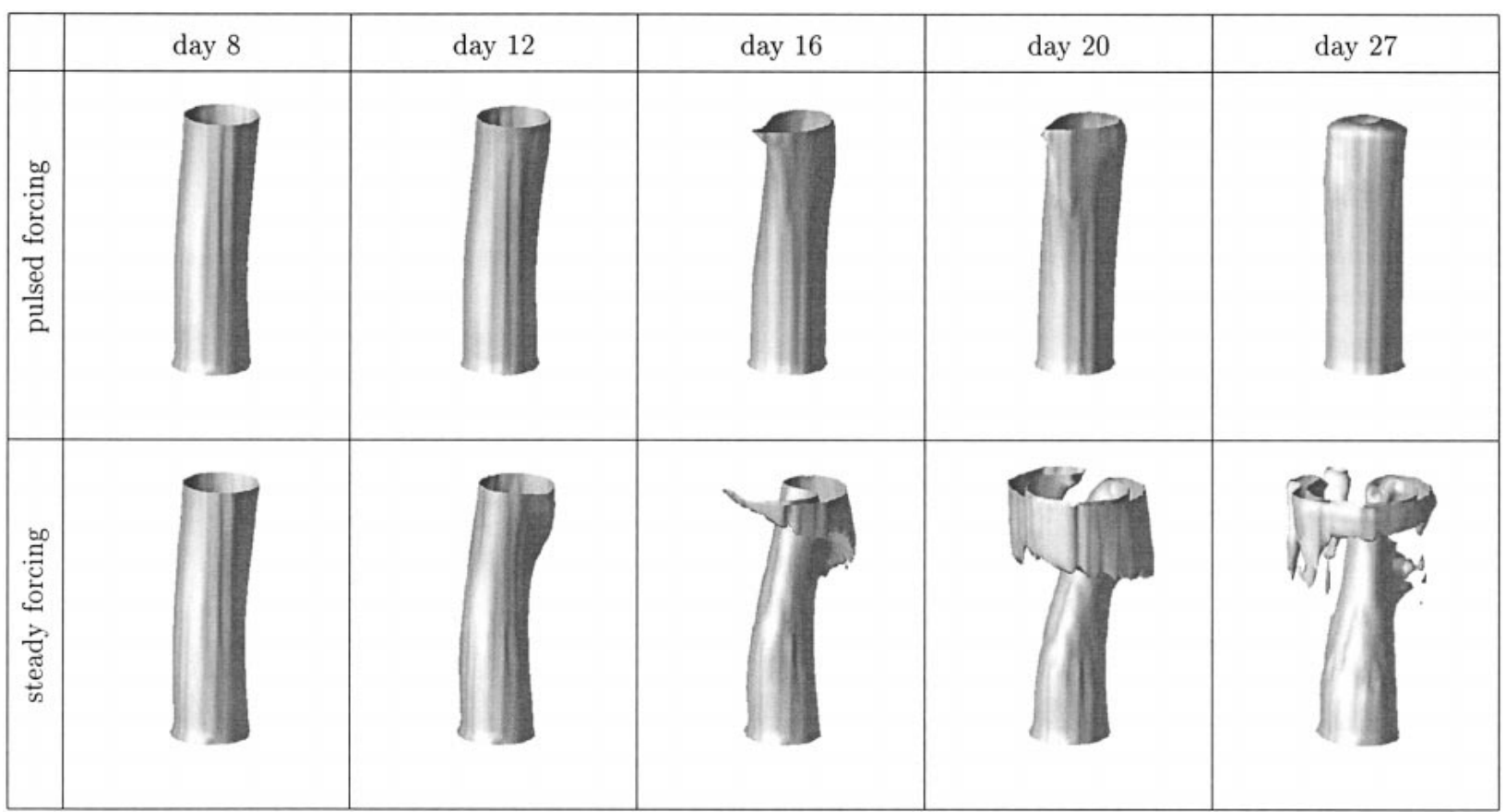

FIG. 9. As in Fig. 5 but for an initial vortex with the smoother PV profile (case SMOOTH, Fig. 8).

effect is observed in the case of steady forcing, illustrated in the bottom row of Fig. 9. Whereas in the case with steeper PV gradients the entire vortex is destroyed (cf. Fig. 5, bottom row), in case SMOOTH the waves only succeed in eroding the top third of the vortex, leaving the lower two-thirds intact. Notice, again, the roll-up of the PV sheet by day 27.

This behavior is particularly surprising in view of the notion of PV "elasticity" proposed by McIntyre (1991). When the dynamics is confined to a single isentropic surface, one can think of the PV gradients as a source of stiffness to quasi-horizontal deformation; steeper PV gradients make quasi-horizontal motions more difficult. On the basis of such arguments, the steep PV gradients surrounding a well-formed polar vortex have been considered responsible for isolating polar air from midlat-
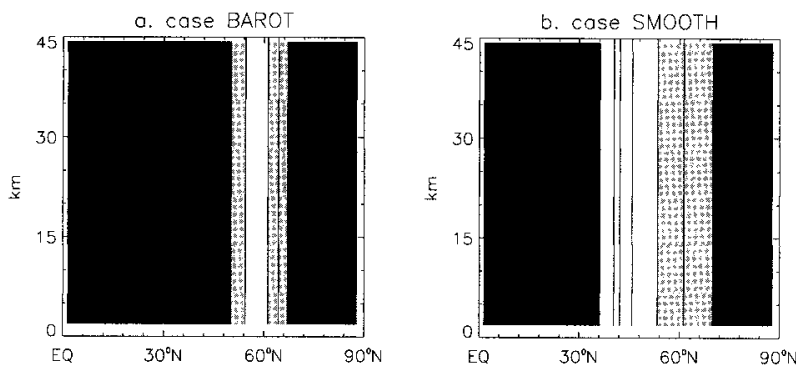

FIG. 10. The nondimensionalized refractive index $n_{0}$, as defined as Eq. (5) for the (a) steep and (b) smoother initial conditions in Figs. 4 and 8 , respectively. Black indicates regions where linear waves are unable to propagate $\left(n_{0}^{2}<1\right)$. Gray indicates regions where $1 \leq n_{0}$ $\leq 3$ and white indicates regions where $n_{0}>3$. itude air, and the vortex edge region (where the PV gradients are the steepest) has been referred to as a "mixing barrier." It may thus appear paradoxical that, in the numerical experiments just described, the case with steeper PV gradients shows a lot more wave breaking than the one with smoother PV gradients.

The resolution to the paradox rests in that PV elasticity arguments fail to take into account the fully threedimensional structure of the polar vortex. The same steep PV gradients that suppress motions in the quasihorizontal direction simultaneously enhance wave propagation in the vertical direction, de facto allowing for a much more violent wave breaking and a stronger disruption of the vortex.

The same conclusion can be arrived at by considering a more traditional tool, the refractive index $n_{0}$ (Matsuno 1970). Although some caution is needed, since the assumptions underlying the notion of refractive index are clearly violated in our experiments (notably the slowly varying nature of the flow), it is nonetheless instructive to plot $n_{0}$ for the two initial condition cases BAROT and SMOOTH. Following Andrews et al. (1987, p. 241), we use the definition

$$
n_{0}^{2} \equiv\left(\frac{a}{2 \pi}\right)^{2}\left(\frac{\bar{q}_{\phi}}{a \bar{u}}-\frac{f^{2}}{4 N^{2} H_{0}^{2}}\right),
$$

where $q$ is the quasigeostrophic potential vorticity, $N^{2}$ the Brunt-Väisälä frequency, and $a$ the radius of the earth. The prefactor $(a / 2 \pi)^{2}$ makes $n_{0}$ nondimensional.

Figure 10a illustrates how the steep PV gradients create a narrow channel that focuses wave activity up the 

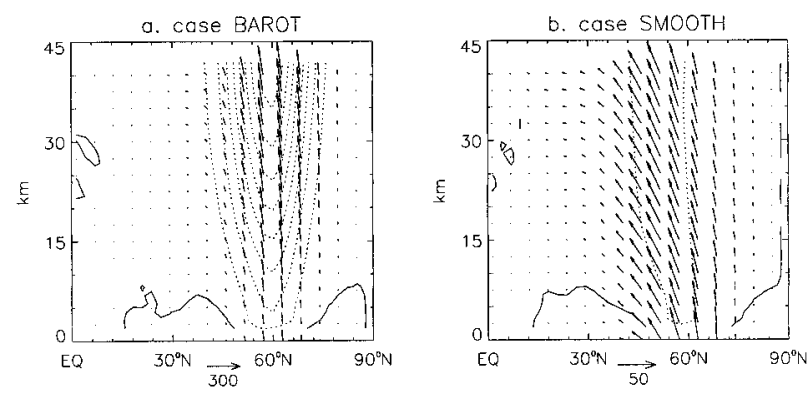

FIG. 11. Eliassen-Palm (E-P) flux vectors superimposed on contours of E-P flux divergence for the (a) BAROT and (b) SMOOTH cases at day 5. The contour interval for E-P flux divergence is $5 \times$ $10^{-6} \mathrm{mPs}^{-2}$ in both cases. The scale of E-P flux vectors for case SMOOTH is six times greater than that for case BAROT.

vortex edge, hence the greater amplification and more vigorous breaking. When the initial PV gradients are smoother the region of wave propagation is much broader, as shown in Fig. 10b. Furthermore, since higher refractive indices are found southward of the polar vortex edge, one expects the Rossby waves to propagate meridionally and equatorward.

This is confirmed by the Eliassen-Palm (E-P) fluxes, shown in Fig. 11, after 5 days of integration. In case BAROT the steep PV gradients channel the waves straight up the vortex, whereas in case SMOOTH considerable meridional propagation can be seen. Note that E-P flux divergence (dotted contours) is much weaker in the SMOOTH case. It should also be noted that the vectors are much smaller in case SMOOTH (the scale is six times greater than in case BAROT); this explains the much smaller impact of the waves on the vortex, which we have already demonstrated. The moral of the story here is that one needs to use caution in extending quasi-horizontal arguments to a situation, such as the one we are considering here, that is intrinsically threedimensional.

\section{b. Forcing amplitude}

In this section we explore how the wave-breaking process is affected by the forcing amplitude $\Phi_{A}$. As in the preceding sections, we limit ourselves to zonal wavenumber $m=1$ forcing. It is worth recalling that DS94, using a quasigeostrophic multilayer contour dynamics model, reported two types of wave-breaking regimes, as mentioned in section 1. One of our objectives here is to determine if similar "remote" and "local" wave-breaking regimes are to be found in our more realistic model.

Consider first the case of pulsed forcing. In Fig. 12, the vortex shape is shown at day 13 , for a sequence of runs identical in every respect but for increasing forcing amplitudes from $\Phi_{A}=200-2000 \mathrm{~m}$. The initial condition is the one shown in Fig. 4, with a steep PV gradient at the vortex edge (case BAROT). Notice how the breaking always originates at the top. Only at the very largest amplitudes can one detect wave breaking originating at the bottom of the vortex. A clear tongue at the vortex bottom is present for $\Phi_{A}=2000 \mathrm{~m}$, and there appears to be one for $\Phi_{A}=1600 \mathrm{~m}$ as well. At these amplitudes, however, the whole vortex has been severely distorted into a "corkscrew"; this is quite unlike the local breaking events reported in DS94, where no breaking occurred in the higher parts of the vortex.

From Fig. 12, one might even be tempted to conclude that the depth of the breaking grows in a linear fashion as the forcing amplitude is increased. A look at the vortex shape at later time, however, reveals that the situation is a bit more complex. In Fig. 13, the vortex is shown at day 30. Since the forcing here is pulsed (it peaks at day 6 and is zero after day 12, cf. Fig. 3), one can speak of a single breaking event; by day 30 the system has become nearly stationary, and one can then observe the results of the wave breaking. At lower amplitudes, the effect of the wave breaking is to erode the upper part of the vortex and, as the amplitude is increased, it appears that the erosion penetrates deeper. Notice, however, that at $\Phi_{A}=1200 \mathrm{~m}$, some substantial vortex "debris" are left in the upper portion of the vortex.

When the forcing is increased further, it is found that only a relatively small portion of the upper vortex is dissipated away; at $\Phi_{A}=1600$ and $2000 \mathrm{~m}$ most of the erosion has occurred in the middle of the vortex (where the narrowest pinching can be seen) while much of the upper vortex, though severely perturbed, has survived. This can be understood as follows: when the wave amplitude is sufficiently strong, the PV gradients are almost completely destroyed in the middle part of the vortex (cf. the pinching in the vortex shape), and consequently the waves have no way of reaching the top, which becomes relatively shielded from the waves.

To validate this scenario more quantitatively, we have computed the vortex area as a function of time and $\theta$. The results, shown in the left column of Fig. 14, were obtained using a box counting method, with the vortex edge defined as SPV $=0.8$. For plotting convenience, we normalize the vortex area with the initial value.

Going down the left column in Fig. 14, that is, as the forcing amplitude is increased, one sees that the location of greatest vortex erosion (cf. the white region on the right portion of the figures) progressively descends. At the largest amplitude more than half of the original vortex area is still present above the $\theta=2000-\mathrm{K}$ isentrope, and the greatest erosion has occurred lower down, between $\theta=1000 \mathrm{~K}$ and $\theta=1500 \mathrm{~K}$. Experiments with steady forcing, shown on the right column of Fig. 14, indicate a similar result, but they are more difficult to visualize since the vortex is severely disrupted in all cases beyond the smallest amplitude.

The preservation of vortex area in the upper levels illustrates the shielding effect of low-level wave breaking, which is also seen in the multilayer contour dynamics models of DS94 and WD99. In essence, vigorous 

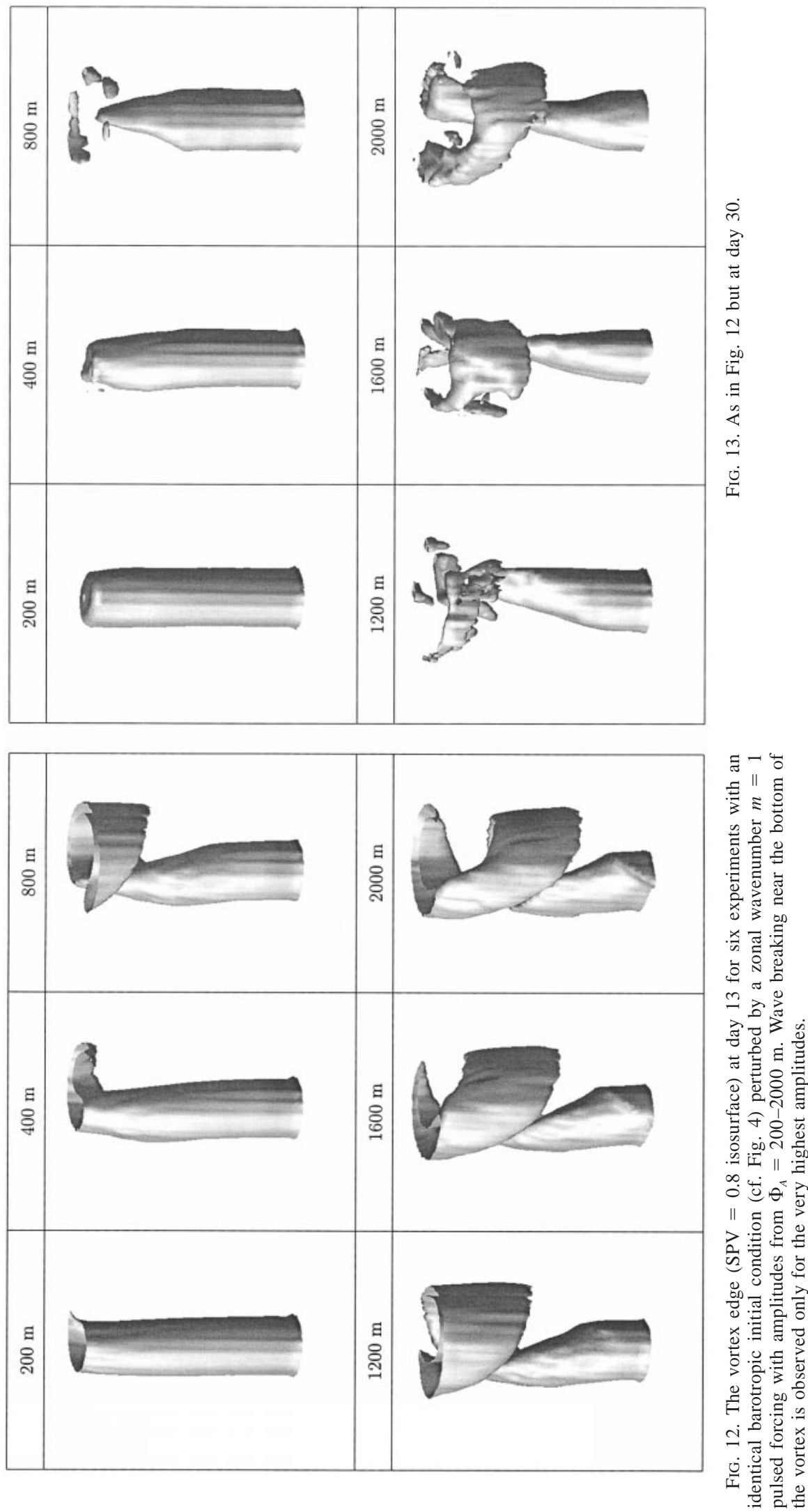


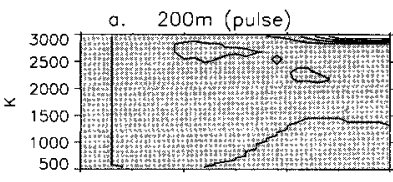

b. $400 \mathrm{~m}$ (pulse)

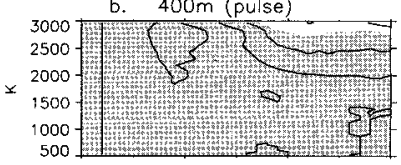

c. $800 \mathrm{~m}$ (pulse)

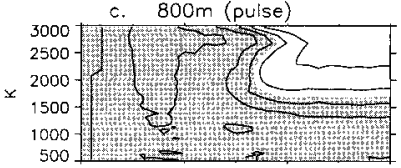

d. $1200 \mathrm{~m}$ (pulse)

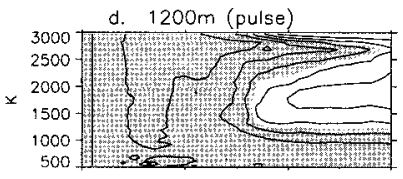

e. $1600 \mathrm{~m}$ (puise)

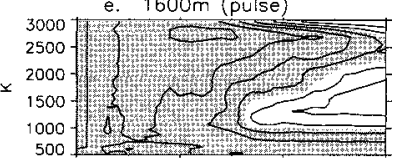

f. $2000 \mathrm{~m}$ (pulse)

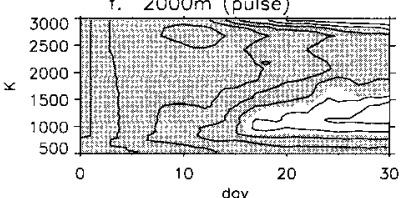

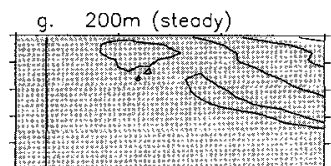

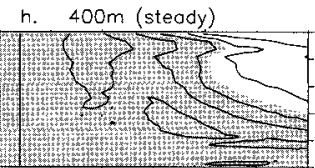

i. $800 \mathrm{~m}$ (steady)

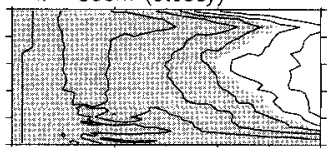

$1200 m$ (stecdy)

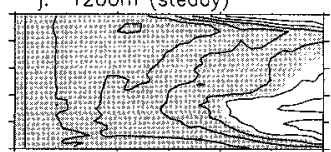

k. $1600 \mathrm{~m}$ (steady)

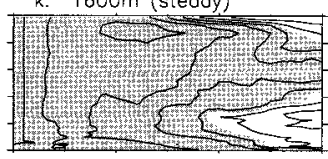

$2000 \mathrm{~m}$ (steady)

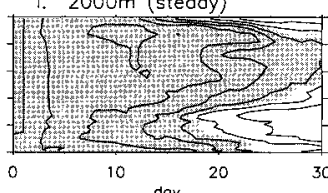

doy
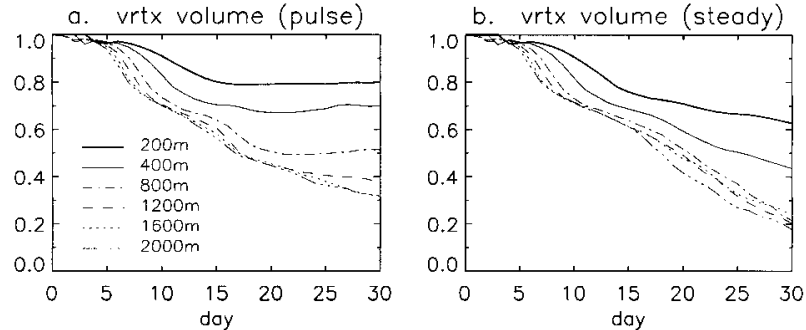

FIG. 15. The time evolution of the vortex volume, normalized by its initial value, for forcing amplitudes from $\Phi_{A}=200-2000 \mathrm{~m}$, for experiments with both (a) pulsed and (b) steady forcing.

$<1200 \mathrm{~m}$. At lower forcing amplitudes, once the wave breaking has occurred, the system becomes very steady, and the erosion is a strong function of amplitude. For the larger amplitudes, however, the system does not seem to become quite as steady (though it might, if we had run our experiments a bit longer); moreover, the amount of erosion after 30 days (typically $60 \%-70 \%$ of the original vortex volume) is only a weak function of forcing amplitude. As we have illustrated earlier (Fig. 13), in the large-amplitude experiments the vortex pinches off at the center, so that the upper portion of the vortex is somewhat shielded from the breaking waves; this could account for the relative insensitivity of the erosion on forcing amplitude once a threshold value is exceeded. The steady forcing experiments (Fig. 15b) show a similar result: except for the weakest forcing amplitudes, roughly $80 \%$ of the original vortex volume is eroded away after 30 days, irrespective of the forcing amplitude. Notice that, in addition, the vortex erosion does not stop since the forcing is not turned off.

\section{Baroclinic vortex}

Having explored the behavior of breaking Rossby waves on barotropic vortices, we now turn our attention to richer initial configurations. Two effects in particular are worth considering: vertical wind shear and the increase of vortex radius with height. The motivation for doing so comes from observations. The climatology compiled by Randel (1992), for instance, shows that the zonal winds at the vortex edge increase several fold between the lower and the upper stratosphere. The study of Mechoso (1990) and, more recently, that of Waugh and Randel (1999) using elliptical diagnostics, show that the mature polar vortex resembles a cone more than a cylinder, especially in the Antarctic.

To determine if and how the vertical wind shear and initial vortex shape affect the breaking of Rossby waves, we have constructed, along the lines of WD99, the three simple initial conditions shown in Fig. 16, for which the parameters are given in Table 1 (see also the appendix for details about how we constructed these initial conditions). Using Randel (1992) as a guideline, case SHEAR has a nearly vertical SPV distribution (Fig. 16a), but the peak winds at the vortex edge vary from erosion. For the experiments with pulsed forcing, Fig. 15a suggests a transition in behavior for $800 \mathrm{~m}<\Phi_{A}$ 

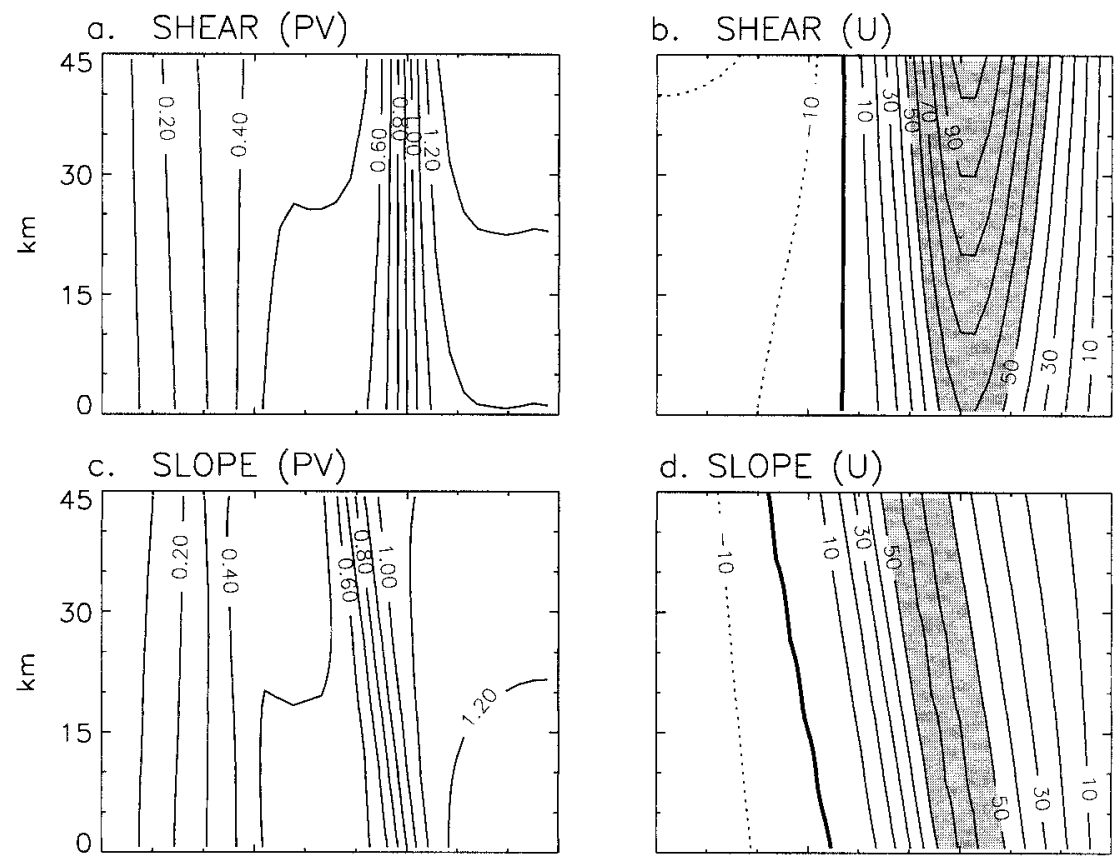

d. SLOPE $(U)$
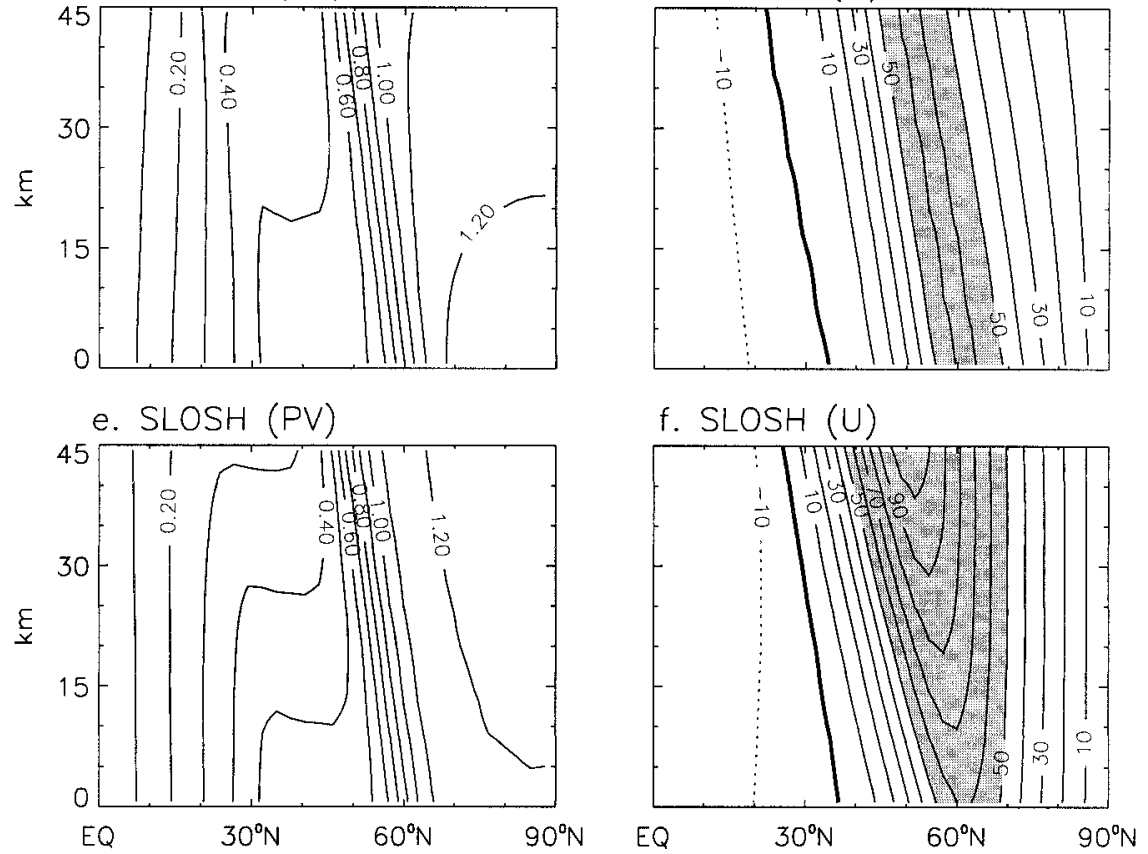

FIG. 16. The initial condition for the cases SHEAR, SLOPE, and SLOSH (cf. Table 1): (left) SPV, (right) zonal winds. The contour intervals are as in Fig. 4, for the reference case BAROT.

$60 \mathrm{~m} \mathrm{~s}^{-1}$ at the bottom of the vortex to roughly $100 \mathrm{~m}$ $\mathrm{s}^{-1}$ at the top (Fig. 16b). Case SLOPE, on the contrary, has constant peak winds of $60 \mathrm{~m} \mathrm{~s}^{-1}$ at all heights (Fig. $16 \mathrm{~d})$, but the vortex edge moves equatorward with height, from $60^{\circ} \mathrm{N}$ at the bottom to $45^{\circ} \mathrm{N}$ at the top (Fig. 16c). Finally, case SLOSH combines both vertical wind shear and a sloping vortex shape (Figs. 16e and 16f). Notice that, in contrast to WD99, for the cases with a conical vortex we have tilted the zero wind line together with the vortex edge, so as to ensure that their latitudinal separation is constant with height. The location of the zero wind line is an important factor in determining the forcing threshold for the onset of wave breaking (Polvani and Plumb 1992).

Consider first experiments with pulsed forcing. The top row of Fig. 17 shows the vortex at day 11 for four experiments with identical forcing with zonal wavenumber $m=1$ and amplitude $\Phi_{A}=800 \mathrm{~m}$. The four experiments only differ in the initial conditions: the left- most is the reference case BAROT (Fig. 4), and the other three are the ones illustrated in Fig. 16. For an initially cylindrical vortex, the comparison of BAROT and SHEAR in Fig. 17 indicates that the vertical wind shear slightly reduces the thickness of the breaking region. A similar conclusion can be drawn by comparing cases SLOPE and SLOSH, for an initially conical vortex. Notice, however, that in either case the effect is rather small especially considering that, in the initial conditions with vertical wind shear, the peak zonal wind at the top of the vortex is nearly double its value at the bottom.

The bottom row of Fig. 17 shows the vortex at day 30 in the four experiments. Since the forcing is turned off after day 12, and the system becomes nearly stationary after day 20, one can assess the effect of a vertical wind shear by considering how much the vortex has been eroded once the breaking event has completed. Comparing cases BAROT and SHEAR, for a cylindrical 


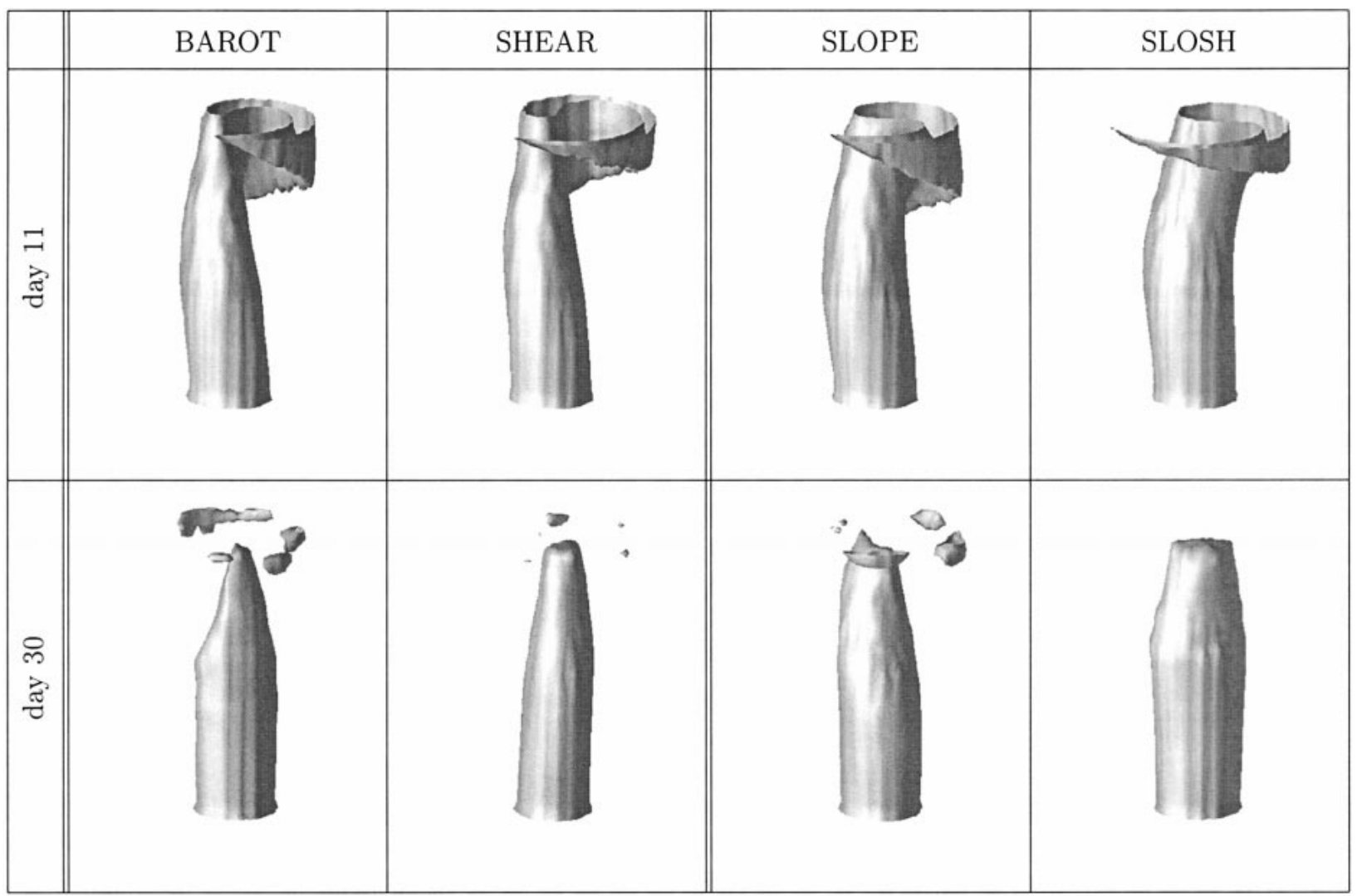

FIG. 17. The vortex edge ( $\mathrm{SPV}=0.8$ isosurface) for four experiments with pulsed forcing $\left(\Phi_{A}=800 \mathrm{~m}, m=1\right)$ using the four initial conditions BAROT, SHEAR, SLOPE, and SLOSH (cf. Table 1). Top row: day 11. Bottom row: day 30.

vortex, and cases SLOPE and SLOSH, for a conical vortex, it appears that when vertical shear is absent the erosion is slightly stronger though, again, the effect is quite minor. Also, comparison of cases BAROT and SLOPE at day 30 tends to suggest that erosion is slightly weaker in the case of an initially conical vortex, though these figures need to be interpreted with caution since the initial vortex area is greater at higher levels in the case of a conical vortex. These results are in agreement with the findings of WD99.

A more rigorous quantification of the vortex erosion is shown in Fig. 18, where the time evolution of the vortex area is plotted for all four cases; the shaded regions highlight vortex areas that are $50 \%$ or greater than the original values (for the corresponding $\theta$ surface). Concentrating first on the rightmost portion of the figures in the left column, the small effect of an initial vertical wind shear can be noticed in that, by day 30 , the shaded region in Fig. $18 \mathrm{~b}$ extends to the $2000-\mathrm{K}$ surface, whereas it only goes up to approximately 1700 $\mathrm{K}$ in Fig. 18a; a similar though smaller effect can be seen by contrasting Figs. 18c and 18d. In the same fashion, the small effect of the initial vortex slope in suppressing the erosion can be seen by contrasting the rightmost portions of Figs. 18a and 18c.

The alert reader, however, might have noticed that the small details we have just pointed to are minor compared to the prominent features in the upper central portion of those figures. From those features, a comparison of Figs. 18a and 18b (and similarly of Figs. 18c and 18d), strongly suggests the opposite conclusion, that is, that the vertical shear enhances the erosion. This is even more dramatically evident in the experiments with steady forcing, illustrated in the right column of Fig. 18. Contrasting Figs. 18e and 18f (and similarly Figs. $18 \mathrm{~g}$ and $18 \mathrm{~h}$ ), one cannot fail to notice that when initial vertical shear is absent (Figs. 18e and $18 \mathrm{~g}$ ) a good fraction of the original vortex area survives, whereas it is entirely wiped out in the experiments with initial vertical shear (Figs. 18f and 18h). The apparent contradiction stems from the fact that these area plots show integral quantities, and thus do not tell the whole story.

A careful look at the isosurfaces reveals what is happening. In Fig. 19 the evolution of a steadily forced vortex with initial condition SHEAR is illustrated at selected times (the corresponding area plot is given in Fig. 18f). By day 15 the Rossby wave has contorted the upper vortex from a cylinder to an annulus of PV. As in the case of the barotropic vortex, the annulus shows vertical alignment over multiple scale heights. This annulus then suffers a secondary instability and rolls up into a number of PV columns so that, at day 18, the 

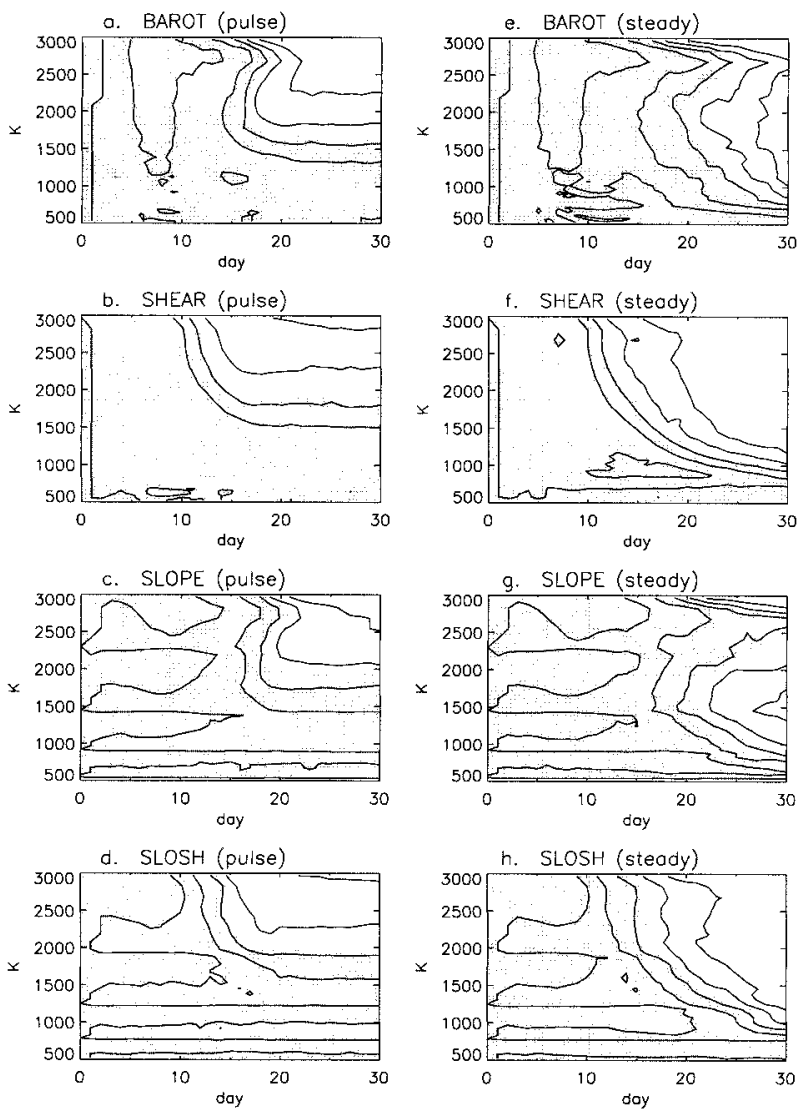

FIG. 18. As in Fig. 14 but for the four different initial conditions BAROT, SHEAR, SLOPE, and SLOSH. In all cases $\Phi_{A}=800 \mathrm{~m}$ and $m=1$.

vortex assumes the appearance of a "chandelier." The vertical wind shear then tilts and stretches the "branches of the chandelier," which rapidly become very thin; our model, being unable to resolve them, dissipates them away. By day 30, the entire upper half of the original vortex has been essentially wiped out.

This evolution should be compared with the corresponding one in the case where no initial vertical wind shear is present (case BAROT, illustrated in the bottom row of Fig. 5). Notice how at day 27, the deep PV columns that result from the roll-up of the PV annulus are mostly untilted. In the absence of wind shear the vortex debris are not stretched subgrid as fast, and thus a larger fraction of the original vortex area survives at upper levels, as seen in Fig. 18e. In the lower levels, however, the vortex "stump" that is left is slightly smaller than in the corresponding case with shear (cf. the shaded regions at day 30 in Figs. 18e and 18f).

Since the dissipation of the vortex debris is controlled by the $\nabla^{8}$ operator in our model, one might ask whether these results are robust to changes in the model resolution. To ascertain this, we have computed several experiments with doubled resolution (T85); this allows us to use a much weaker hyperviscosity (the dissipation acts mostly around wavenumber 85 , instead of 42 ). Figure 20 shows the evolution of the vortex areas for initial conditions BAROT and SHEAR at T85 resolution with steady forcing parameters $\Phi_{A}=800 \mathrm{~m}$ and $m=1$; the corresponding T42 evolutions are in Figs. 18e and 18f. From these figures and the corresponding isosurface plots (not shown), it can be deduced that the qualitative results are identical at both resolutions, although the vortex area dissipation is noticeably weaker at T85.

To conclude and summarize this section, the vortex volumes for the four cases BAROT, SHEAR, SLOPE, and SLOSH are plotted in Fig. 21 for both pulsed and steady forcing $\left(\Phi_{A}=800 \mathrm{~m}\right.$ and $\left.m=1\right)$. The main result is that, in spite of substantial differences in these four initial conditions, the wave-breaking process differs little in terms of the volume that remains in the vortex after the wave breaking, and the most notable effect is that of the vertical wind shear in accelerating the stretching and thus the dissipation of the vortex debris. We have verified that these same conclusions hold at higher forcing amplitudes by repeating the pulsed forcing experiments with $\Phi_{A}=1600 \mathrm{~m}$.

\section{Wavenumber-2 forcing}

All experiments discussed thus far have considered the response of the polar vortex to forcing with zonal wavenumber $m=1$. In this section, we look at the response to zonal wavenumber $m=2$, which plays an important role in stratospheric sudden warming events. Typically, such events are associated with a rapid increase in zonal-mean stratospheric temperatures and, on occasion, by a reversal of the zonal-mean winds from westerlies to easterlies (Andrews et al. 1987). Sudden warmings are often characterized by a splitting of the polar vortex, corresponding to $m=2$ forcing (as shown in Fig. 2).

We first consider the simplest case, that is, the response of a barotropic vortex to a pulsed forcing with zonal wavenumber $m=2$ and amplitude $\Phi_{A}=800 \mathrm{~m}$. The evolution of the vortex edge for this case is shown in the top row of Fig. 22. Instead of deforming into a comma shape, the vortex assumes an elliptical configuration (day 5), as one would expect, and soon develops two cuspy regions near the top (day 10). The wave breaking thus strips two (symmetric) filaments from the vortex (days 15 and 20), and the final result is an eroded vortex not unlike the one in the corresponding $m=1$ case.

Notice, however, that the erosion is much weaker than in the $m=1$ case with identical initial condition and forcing amplitude (top row of Fig. 5). This is quantified in Figs. 23a and 23b, where we have plotted zonal-mean wind and the temperature evolution associated with this $m=2$ breaking event; this figure should be contrasted with its $m=1$ counterpart (Fig. 6). The evolution of vortex area (Fig. 24a) shows similar behavior, with some vortex erosion occurring at upper levels, but in general 


\begin{tabular}{|l|c|c|c|c|}
\hline day 9 & day 11 & day 13 & day 15 & day 17 \\
\hline & & & \\
\hline
\end{tabular}

FIG. 19. The vortex edge, at selected times, for initial condition SHEAR perturbed with a steady forcing $\left(\Phi_{A}=800 \mathrm{~m}, m=1\right)$. The top row shows the SPV $=0.8$ isosurface. For clarity, the bottom row shows the SPV $=0.95$ isosurface and has been rotated $20^{\circ}$ clockwise about the pole.

much weaker in comparison with the $m=1$ case (Fig. $14 \mathrm{c})$. In sum, then, a cylindrical barotropic vortex subject to $m=2$ forcing seems to produce a rather weak simulation of sudden warmings, and no real splitting of the vortex.

A first idea would be that the 800-m forcing amplitude we have used is not sufficiently large. Exploring this avenue, we show in the bottom row of Fig. 22 the results of an experiment with $\Phi_{A}=1200 \mathrm{~m}$ (and $m=2$ ). Notice the dramatic difference from the weaker amplitude case. The early elliptical deformation (day 5) soon turns into an " $S$ " shape (day 10), as one would expect from vortex dynamics. The three-dimensional structure then becomes very interesting, as the wave rips the vortex into two helical PV tongues (day 15), each spanning almost $180^{\circ}$ of longitude and tilting westward with height (not surprisingly given that these are Rossby waves). The two helical tongues eventually pinch off the main vortex (day 20), and the final result of this event is a pair of rather elongated and vertically aligned corotating vortices in the top third of the model hovering over a columnar vortex in the lower two-thirds (day 30). This splitting of the vortex into two parts is not unlike the observations.

We have performed experiments at even larger forcing amplitudes as well as with steady forcing. In those cases (not shown) the two helical PV tongues can be seen to wind down from the top over a full $360^{\circ}$ lati- tudinal span. Unfortunately, we are unable to cite observational evidence for such helical structures accompanying sudden warming events. Furthermore, a quick glance at Figs. 1 and 2 shows that the peak amplitudes of eddy geopotential near the tropopause are similar during strong $m=1$ and $m=2$ events (roughly 500 $\mathrm{m})$. Hence, it may be difficult to defend why much larger amplitudes would be required to produce sudden warmings as opposed to comma shapes. However, another possibility can be explored.

A second idea for producing strong $m=2$ events without increasing the forcing amplitude is suggested by observational studies that have shown how, in the case of many sudden warming events, a wavenumber-2 pulse is preceded by a wavenumber-1 pulse (e.g., Labitzke 1981). This $m=1$ pulse is said to "precondition" the polar stratosphere for a subsequent sudden warming event. Evidence for such preconditioning is also found in model simulations of sudden warming events (e.g., Butchart et al. 1982). This motivates us to consider the effects of preconditioning on the response to $m=2$ forcing.

We use the very first experiment discussed in section 3 , the barotropic vortex subject to a pulsed wavenumber-1 forcing (Fig. 5, upper panel), as a crude representation of preconditioning. The end state of this experiment after 30 days of integration serves as the initial condition for a new experiment with steady 800-m 

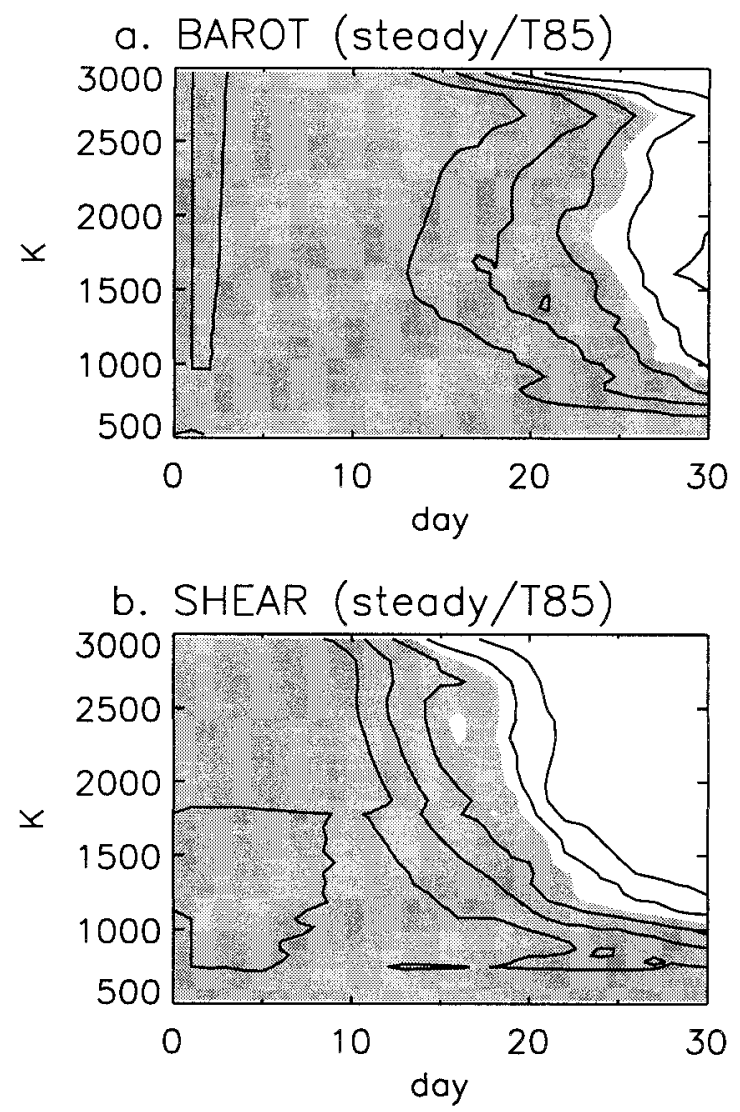

FIG. 20. As in Figs. 18e and $18 \mathrm{f}$ but for two experiments at T85 resolution.

wavenumber-2 forcing, referred to as the "preconditioned $m=2$ " experiment.

The preconditioned vortex has a very marked conical shape, as can be seen in the 800-m frame of Fig. 13. This conical shape is a result of the erosion from the $m$ $=1$ event. The corresponding zonal-mean PV shows a gradient tilting poleward with height (Fig. 25a). ${ }^{2}$ Similarly, the initial zonal-mean zonal wind shows a poleward shift with height of the polar night jet (Fig. 25b). This is an important characteristic of preconditioning.

The results of an experiment initialized with the preconditioned vortex of Fig. 25 and forced with an $m=$ 2 wave with amplitude $\Phi_{A}=800 \mathrm{~m}$, are shown in the bottom row of Fig. 23. The zonal-mean zonal wind in the preconditioned $m=2$ experiment shows strong deceleration, with easterlies forming in the upper levels (Fig. 23c). This is accompanied by a significant increase in the zonal-mean temperatures (Fig. 23d). The vortex

\footnotetext{
${ }^{2}$ We also note, en passant, that the $m=1$ wave-breaking event has produced a second set of strong PV gradients in the subtropics, located about $20^{\circ} \mathrm{N}$ and rather well vertically aligned above $1000 \mathrm{~K}$. Similar "subtropical barriers" can be produced in one-layer models (e.g., Polvani et al. 1995), but we can here see its full vertical extent.
}
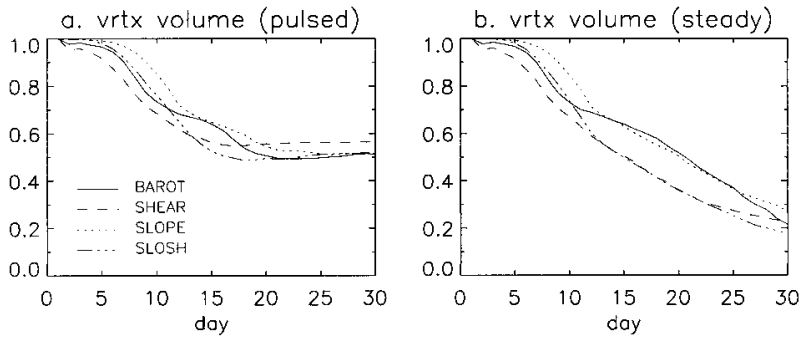

FIG. 21. The time evolution of the vortex volume, for forcing cases BAROT, SHEAR, SLOPE, and SLOSH for experiments with $\Phi_{A}=$ $800 \mathrm{~m}$ and $m=1$, with both (a) pulsed and (b) steady forcing.

area diagnostic (Fig. 24) shows that preconditioning significantly increases the vortex erosion associated with $m=2$ forcing, with the vortex losing much of its initial area in the mid- and upper levels very rapidly between day 10 and day 15 . Overall, our crude representation of preconditioning appears to have dramatically amplified the wave breaking associated with wavenumber-2 forcing.

Theoretical models of stratospheric sudden warmings have traditionally been formulated in the context of atmospheric flows that are smoothly varying in the meridional direction. For example, the model of Matsuno (1971) is based upon the scenario of Rossby waves propagating vertically on a smooth climatological background state. The "self-tuned resonant cavity instability" mechanism of Plumb (1981) relies upon a zonal channel with sidewalls to confine waves in the meridional direction.

Our experiments with $m=2$ forcing provide an alternative view of sudden warmings in a scenario where there are sharp meridional gradients in the $\mathrm{PV}$ associated with a well-formed polar vortex. The PV discontinuity at the vortex edge provides a mechanism for trapping waves in the meridional direction. It is then the vertical structure of the vortex, rather than a smoothly varying refractive index in the horizontal direction, that controls wave propagation. In this view of sudden warmings (McIntyre 1982), preconditioning acts to reduce the vortex area in the upper levels and make the vortex slope inward (Fig. 25). This decrease in vortex area with increasing height tends to "focus" the upward propagating waves toward the pole.

\section{Discussion}

The study of stratospheric circulation has benefited tremendously from the conceptual framework provided by two-dimensional vortex dynamics (e.g., McIntyre and Palmer 1983, 1984; Juckes and McIntyre 1987; Polvani and Plumb 1992). Two-dimensional concepts such as the filamentation of vortices in straining flows and the roll-up of filaments have an obvious and direct application to stratospheric wave breaking. However, the wintertime polar stratospheric vortex is an inherently three-dimensional object. Much of the wave breaking 


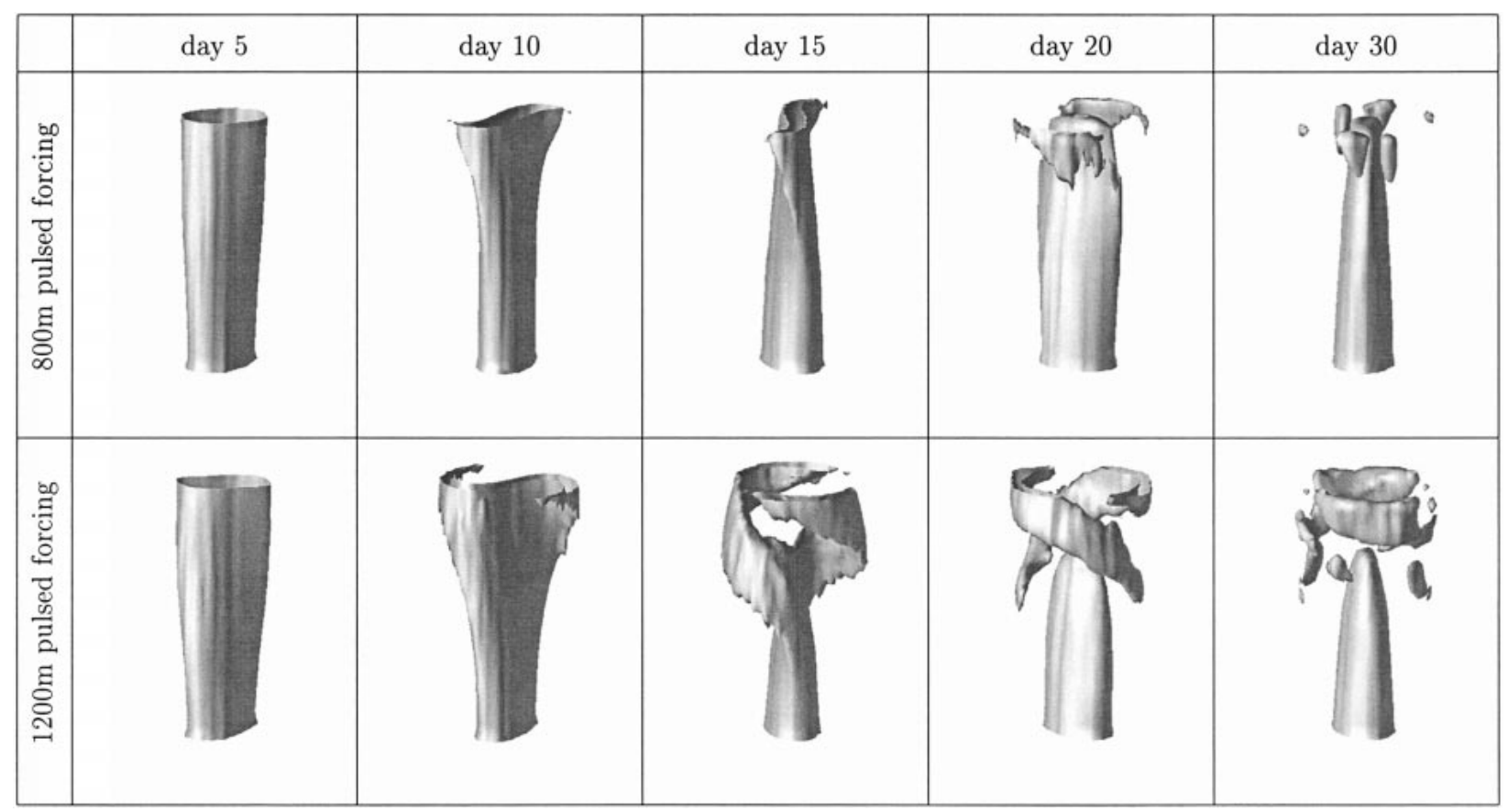

FIG. 22. The vortex edge ( $\mathrm{SPV}=0.8$ isosurface) for $m=2$ pulsed forcing with amplitudes $\Phi_{a}=800 \mathrm{~m}$ (top row) and $\Phi_{a}=1200 \mathrm{~m}$ (bottom row). The initial condition is case BAROT (cf. Table 1 and Fig. 4).

that occurs in the polar stratosphere is associated with vertical propagation of Rossby waves-a process that clearly lies outside the domain of two-dimensional vortex dynamics.

In this study we have tried to explore the parameter space of three-dimensional vortex dynamics, with the hope of furthering our understanding of the stratospheric circulation. Our exploration has highlighted the following three-dimensional properties of Rossby wave breaking: (i) the deep vertical structure of the PV tongue associated with a wavenumber-1 breaking event; (ii) the roll-up of the PV tongue into vertically coherent columns and the subsequent tilting of the columns by vertical shear; (iii) enhanced wave penetration and breaking associated with a sharper meridional PV gradient; (iv) the focusing/defocusing of vertically propagating wave activity due to changes in vortex area with height; (v) the shielding effect associated with low-level wave breaking. Each of the above properties has significant implications for stratospheric dynamics and will be discussed in detail below. Several of these properties were also noted in the multilayer quasigeostrophic contour dynamics studies of DS94 and WD99. This gives us the confidence that they are indeed robust.

When a three-dimensional barotropic vortex is forced by wavenumber- 1 bottom topography, wave breaking is initiated near the top. This event is characterized by the well-known comma shape exhibited by a two-dimensional vortex embedded in a wavenumber- 1 straining flow. The contour dynamics study of DS94 showed that the filaments associated with the comma shape are ver- tically aligned for an initially barotropic vortex. WD99 showed that this is true even for an initially baroclinic vortex. We see the same kind of vertical alignment in our primitive equation model. Furthermore, the contour advection study of Schoeberl and Newman (1995) also found evidence for deep filamentary structures in the observations. All of this indicates a strong tendency for large-scale PV structures to remain vertically aligned. Evidence for this alignment mechanism may also be found in studies of multilayer quasigeostrophic vortex dynamics (Polvani 1992; Sutyrin et al. 1998). However, smaller-scale PV structures-such as those formed by the roll up of the vertically aligned filaments-appear to be more susceptible to tilting by the background vertical shear.

McIntyre and Palmer (1984) speculated that there was a local dynamical instability associated with the PV tongue extruded from the polar vortex, based upon their coarse-grained isentropic PV analysis. Indeed, in our experiments, the deep PV tongue rolls up into vertical columns. These PV columns are quite tall-extending over two to three scale heights - and are initially upright, even in cases with background vertical shear. The roll-up appears to be an essentially barotropic process, analogous to the roll-up of filaments in two-dimensional vortex dynamics. Stretching this analogy, one may conclude that these coherent rolled-up vortices would also be quite resistant to further mixing, as is the case in two-dimensional turbulence. However, any vertical shear in the background flow quickly tilts and stretches these PV columns, providing a uniquely three-dimen- 

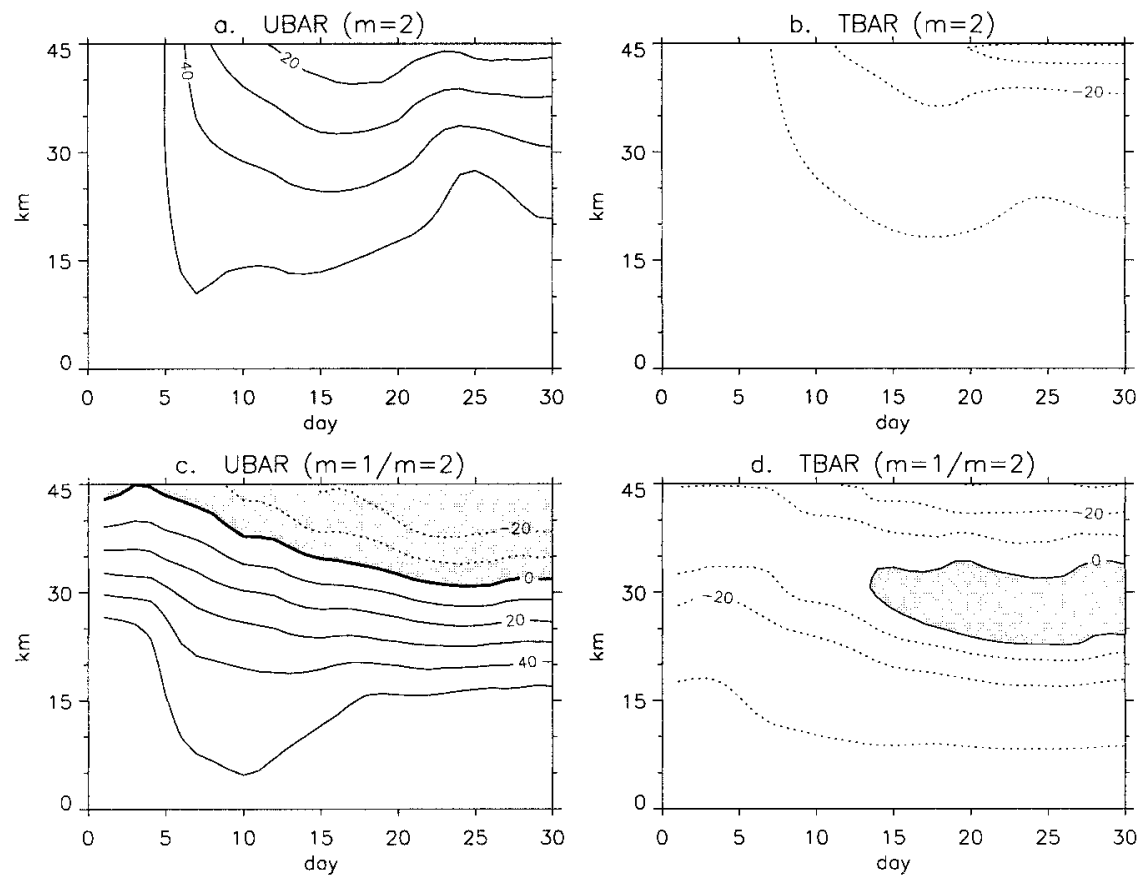

FIG. 23. As in Fig. 6 but for (a), (b) the barotropic $m=2$ experiment, and for (c), (d) the preconditioned $m=2$ experiment.

sional mechanism for dissipating the columns. Background vertical shear may therefore be far more effective at dissipating coherent vortical structures than horizontal straining — a fact that has important implications for the scaling properties of tracer mixing (Haynes and Anglade 1997). Although vertical shear accelerates the rate of vortex erosion after the roll-up has occurred, its effect on the total amount of vortex erosion is quite small. The total amount of material that is eroded from the vortex is determined by the initial filamentation process, which shows only weak sensitivity to vertical shear.

It is often argued that Rossby wave elasticity associated with the polar vortex edge acts as a barrier that shields the inside of the vortex from the vigorous turbulent mixing, which takes place just outside the vortex edge (e.g., Juckes and McIntyre 1987). This would im-
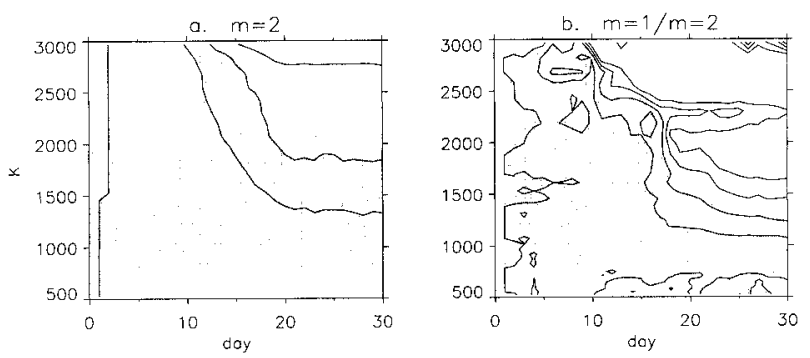

FIG. 24. The time $\theta$ evolution of vortex area, normalized by its initial value, for (a) the barotropic $m=2$ experiment, and (b) and the preconditioned $m=2$ experiment. Plotting conventions as in Fig. 14. ply that the sharper the vortex edge, the more effective it would be as a mixing barrier. This argument, based upon two-dimensional vortex dynamics, fails to take into account the effects of vertical wave propagation. As evident in our experiments, the sharper the vortex edge, the easier it is for Rossby waves to propagate vertically along that edge. Thus a sharper vortex edge can actually lead to increased wave breaking and mixing in the upper levels.

The three-dimensional shape of the polar vortex has a significant effect on the amplitude of wave breaking, as first suggested by McIntyre and Palmer $(1983,1984)$. An increase in vortex area with height can "defocus" the wave activity away from the pole and reduce the amplitude of wave breaking (WD99). A decrease in vortex area with height, on the other hand, can lead to a "focusing" of wave activity toward the pole and amplification of wave breaking. Our experiments show that an initial wave breaking event can cause vortex erosion near the top, decreasing the area of the vortex and making it more prone to disruption by subsequent wavebreaking events. This provides a geometric interpretation of the preconditioning associated with stratospheric sudden warming events.

For large topographic forcing amplitudes, the polar vortex is severely disrupted at the lower levels. This destroys the strong meridional PV gradient that allows waves to propagate vertically along the vortex edge, shielding the regions above from further wave propagation. Thus, the amplitude of upper-level wave breaking is reduced as the lower-level wave breaking increas- 

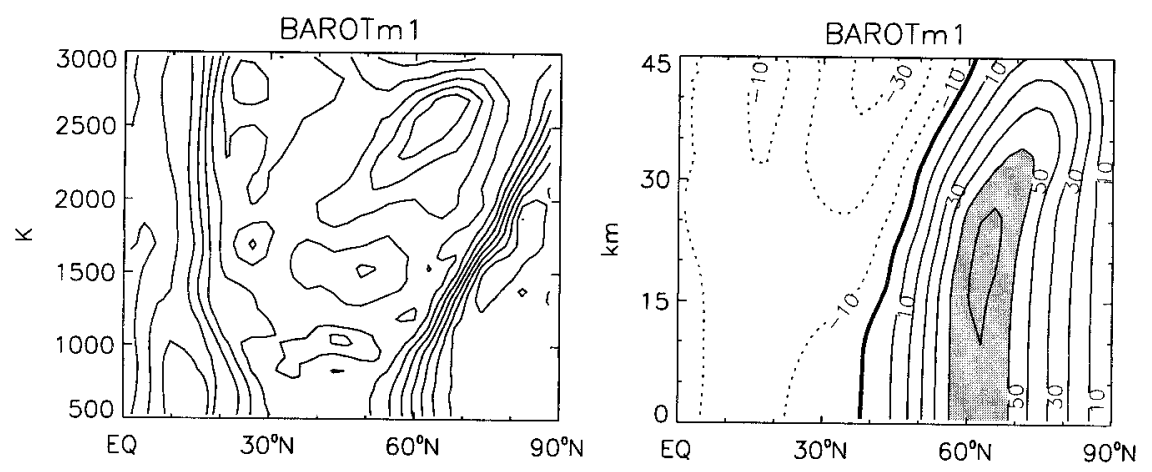

FIG. 25. The initial condition for the preconditioned $m=2$ forcing experiment. (a) The zonalmean SPV at day 30 for a barotropic vortex subject pulsed forcing with $m=1$ and $\Phi_{A}=800 \mathrm{~m}$ (case BAROT). (b) The corresponding zonal-mean zonal wind. The corresponding isosurface can be seen in the 800-m frame of Fig. 13. Plotting conventions as in Fig. 4.

es. This shielding effect was noted in the multilayer contour-dynamics modeling studies of DS94 and WD99. In our primitive equation model experiments, however, we find that the transition occurs much more gradually as the forcing amplitude is increased. Furthermore, at very large forcing amplitudes where local breaking is observed (cf. Fig. 12 at $2000 \mathrm{~m}$ ) the upper half of the vortex is severely disturbed, while in the multilayer contour-dynamics experiments it is relatively intact. This discrepancy can perhaps be attributed to the way in which the forcing is applied in our model (in which we perturb the bottom as a material surface). Another difference between our experiments and those of WD99 is the much smaller sensitivity we find to the initial vortex shape and vertical wind shear. This suggests that the surprising sensitivity reported by WD99 may be more of a peculiarity of the contour-dynamics model than an important realistic effect.

In summary, our experiments demonstrate that looking at the evolution of a well-formed polar vortex with sharp PV gradients provides a rich, nonlinear view of stratospheric dynamics - a view that is strikingly different from a more traditional description of vertically propagating linear waves on a smoothly varying basic state. The numerical experiments in this study have considered rather idealized scenarios of the stratospheric circulation and provide mostly qualitative insights. More realistic experiments and case studies are needed to make the quantitative connection between the properties of three-dimensional vortices and the stratospheric circulation.

Acknowledgments. We are grateful to Alan Plumb and Bill Randel for enlightening discussions, to Darryn Waugh and David Dritschel for sharing their results with us prior to publication, to Aimé Fournier and Mark Taylor for a careful reading of the manuscript, and to Isaac Held for originally inspiring this work. This work was supported, in part, by an NSF grant to Columbia University and through the NSF sponsorship of NCAR.

\section{APPENDIX Vortex Initialization}

We wish to initialize our simulations with a specified axisymmetric vortex shape. We use the subscripts $v, z$, $s$, and $e$ to denote the vortex edge, the zero wind line, the southern edge of the surf zone, and the equator, respectively. The vortex edge is characterized by a peak zonal velocity $U_{v}$ and latitudinal location $\phi_{v}$. We assume that outside the vortex, there is a surf zone extending out to latitude $\phi_{s}$, and that the zero wind line lies at a latitude $\phi_{z}$, such that $\phi_{v}>\phi_{z}>\phi_{s}$. The zonal velocity at the equator is denoted by $U_{e}$.

To determine the initial velocity and temperature distribution, we use the following approach: we write down an analytical expression for the absolute vorticity $\zeta$ with five unknown parameters. These are determined by applying matching conditions requiring continuity of the absolute angular momentum and its meridional derivative at $\phi_{s}$, in addition to the constraints on the zonal velocity at the vortex edge, the zero wind line and at the equator listed above. Once the distribution of $\zeta$ is known, we can easily compute the zonal velocity $U$ at each level and latitude. Finally, we compute the temperature distribution by requiring that the flow be in thermal wind balance, using the assumptions that the meridional velocity $V \equiv 0$ and that the model levels coincide with constant pressure levels.

The analytical expression for $\zeta$ is chosen as follows. For a Northern Hemispheric vortex, we let $\zeta$ have a step-function-like profile at the vortex edge $\left(\phi=\phi_{v}\right)$, with a nearly constant value up to the surf zone edge $\left(\phi=\phi_{s}\right)$, a cubic profile in $\mu$ from the surf zone edge to the equator, and a solid body rotation profile in the Southern Hemisphere:

$$
\zeta= \begin{cases}A S(\mu)+B, & \text { for } \mu_{s} \leq \mu, \\ C \mu+D \mu^{3}, & \text { for } 0 \geq \mu \geq \mu_{s}, \\ E \mu, & \text { for } \mu \leq 0,\end{cases}
$$

where "step function" $S$ is defined as 


$$
\mathcal{S}(\mu) \equiv \frac{1}{2}\left\{1+\tanh \left(\frac{\mu-\mu_{v}}{\Delta \mu}\right)\right\},
$$

where $\mu \equiv \sin (\phi), \Delta \mu=\Delta \phi \cos \phi, \phi$ is the latitude, $\Omega$ is the angular velocity of planetary rotation, and $a$ denotes the planetary radius. The parameters $A, B, C$, $D$, and $E$ are yet to be determined.

With regard to the three-dimensional structure of the vortex, we assume that $U_{v}, \phi_{v}, \phi_{z}$, and $\phi_{s}$ are linear functions of log-pressure height $z$ :

$$
\begin{aligned}
& U_{v}=U_{v b}+\left(U_{v t}-U_{v b}\right) \frac{z}{H}, \\
& \phi_{v}=\phi_{v b}+\left(\phi_{v t}-\phi_{v b}\right) \frac{z}{H}, \\
& \phi_{z}=\phi_{z b}+\left(\phi_{v t}-\phi_{v b}\right) \frac{z}{H}, \\
& \phi_{s}=\phi_{s b}+\left(\phi_{v t}-\phi_{v b}\right) \frac{z}{H},
\end{aligned}
$$

where $H$ is the model height, $U_{v b}$ denotes the vortex edge zonal velocity at the bottom of the vortex, $U_{v t}$ the vortex edge zonal velocity at the top, $\phi_{v b}$ the vortex edge latitude at the bottom, $\phi_{v t}$ the vortex edge latitude at the top, $\phi_{z b}$ the zero wind latitude at the bottom, and $\phi_{s b}$ the edge of the surf zone at the bottom. Note that (A5) and (A6) have the same vertical variation as (A4), which means that $\phi_{z}-\phi_{v}$ and $\phi_{s}-\phi_{v}$ are independent of $z$. We further assume that $U_{e}$ and $\Delta \phi$ are independent of $z$. Table 1 shows the vortex parameter values for the different cases; in all cases $\phi_{z}=\phi_{v}-23^{\circ}, \phi_{s}=\phi_{v}$ $-25^{\circ}$, and $U_{e}=-20 \mathrm{~m} \mathrm{~s}^{-1}$.

Note that we have assumed the step-function-like meridional profile (A2) for the absolute vorticity, not the PV. It turns out this is sufficient to produce a step function meridional profile for the PV at approximately the same latitudinal location, thus obviating the need for carrying out a complex three-dimensional inversion of PV. Since we are only interested in generating idealized vortex shapes as initial conditions for our integrations, a more accurate three-dimensional PV inversion procedure would not provide any advantage.

In spherical geometry, $\zeta$ for zonally symmetric flows may be written as

$$
\zeta=2 \Omega \mu-\frac{1}{a} \frac{\partial}{\partial \mu} U \cos \phi .
$$

The absolute angular momentum (per unit mass) $M$ may then be computed by integrating (A7) to the North Pole:

$$
M \equiv \Omega a^{2} \cos ^{2} \phi+U a \cos \phi=a^{2} \int_{\mu}^{1} d \mu \zeta .
$$

We use (A8) and the vortex parameters to compute the absolute angular momentum at the vortex edge, at the zero wind line, and at the equator:

$$
\begin{aligned}
& M_{v}=\Omega a^{2}\left(1-\mu_{v}^{2}\right)+U_{v} a \cos \phi_{v}, \\
& M_{z}=\Omega a^{2}\left(1-\mu_{z}^{2}\right), \\
& M_{e}=\Omega a^{2}+U_{e} a .
\end{aligned}
$$

It is useful to define the integral of (A2) as

$$
\begin{aligned}
\mathcal{R}(\mu) \equiv & \int_{\mu}^{1} d \mu S \\
= & \frac{1-\mu}{2} \\
& +\frac{\Delta \mu}{2} \ln \left\{\frac{\exp \left(\frac{1-\mu_{v}}{\Delta \mu}\right)+\exp \left(-\frac{1-\mu_{v}}{\Delta \mu}\right)}{\exp \left(\frac{\mu-\mu_{v}}{\Delta \mu}\right)+\exp \left(-\frac{\mu-\mu_{v}}{\Delta \mu}\right)}\right\} .
\end{aligned}
$$

Assuming that $\phi_{z}>\phi_{s}$, we can integrate (A1) to the North Pole and use the expressions [(A9), (A10)] for the angular momentum to solve for the parameters $A$ and $B$ to obtain

$$
\begin{aligned}
A & =\frac{a^{-2} M_{v}-B\left(1-\mu_{v}\right)}{\mathcal{R}_{v}}, \\
B & =a^{-2} \frac{M_{z}-M_{v} \frac{\mathcal{R}_{z}}{\mathcal{R}_{v}}}{\left(1-\mu_{z}\right)-\frac{\mathcal{R}_{z}}{\mathcal{R}_{v}}\left(1-\mu_{v}\right)} .
\end{aligned}
$$

Having obtained $A$ and $B$, one may next compute the angular momentum at the edge of the surf zone, $M_{s}$, by integrating (A1)

$$
M_{s}=a^{2}\left\{A \mathcal{R}\left(\mu_{s}+B\left(1-\mu_{s}\right)\right\} .\right.
$$

We then integrate (A1) from the equator to the edge of the surface zone $\left(\phi=\phi_{s}\right)$, and require continuity of $M$ and $\zeta$ to obtain the following expressions for parameters $C$ and $D$ :

$$
\begin{aligned}
& C=\left(A \mathcal{S}_{s}+B\right) \mu_{s}^{-1}-D \mu_{s}^{2}, \\
& D=2\left(A \mathcal{S}_{s}+B\right) \mu_{s}^{-3}-4 a^{-2}\left(M_{e}-M_{s}\right) \mu_{s}^{-4} .
\end{aligned}
$$

Finally, the requirement that $U=U_{e}$ at the equator and the assumption of solid body rotation in the Southern Hemisphere gives us the value of parameter $E=2(\Omega$ $\left.+a^{-1} U_{e}\right)$.

Once the parameters $A, B, C, D$, and $E$ are known, we use (A8) to compute the meridional profile of angular momentum $M$ by integrating (A1) in the meridional direction 


$$
M= \begin{cases}a^{2}\{A \mathcal{R}(\mu)+B(1-\mu)\}, & \text { for } \mu_{s} \leq \mu, \\ M_{e}-a^{2}\left\{\frac{C}{2} \mu^{2}+\frac{D}{4} \mu^{4}\right\}, & \text { for } 0 \geq \mu \geq \mu_{s}, \\ M_{e}-a^{2} \frac{E}{2} \mu^{2}, & \text { for } \mu \leq 0 .\end{cases}
$$

From this, the zonal velocity $U$ can be determined using (A8).

We assume that the meridional velocity $V \equiv 0$, and that all model levels are also constant pressure levels. This assumption allows us to easily balance the zonal velocity with the temperature. We define the temperature perturbation $T^{\prime}$ as deviations from the global mean reference temperature $T_{0}=239.14 \mathrm{~K}$, which corresponds to a reference scale height $H_{0}=7 \mathrm{~km}$. Denoting by $\Phi^{\prime}$ the deviations of the geopotential $\Phi$ from the globally averaged value at each level, we may write the hydrostatic equation as

$$
\frac{\partial \Phi^{\prime}}{\partial \ln p}=-R T^{\prime} .
$$

The meridional gradient of $\Phi^{\prime}$ on constant pressure surfaces may be obtained from the tendency equation for $V$ as

$$
\frac{1}{a} \frac{\partial \Phi^{\prime}}{\partial \phi}=-U\left(2 \Omega \mu+\frac{U}{a} \tan \phi\right) .
$$

Note that relationship (A20) is only valid when the model levels initially coincide with constant pressure levels; this relationship will not be valid in the general case of sigma model levels with arbitrary bottom topography.

To obtain a balanced initial condition, all that remains now is to determine the surface perturbation geopotential $\Phi_{\text {surf }}^{\prime}$ and the $T^{\prime}$ values at each model level. We integrate (A20) in the meridional direction to compute $\Phi^{\prime}$ at each half-level of the model (i.e., midway between the actual model levels) and also at the surface and at the top of the model, using the constraint that the global average of $\Phi^{\prime}$ is zero. We then invert the hydrostatic relation (A19) to compute $T^{\prime}$ values at all the model levels. Note that by applying (A20) at the half-levels we avoid the problem of the two-grid wave in the vertical (e.g., see Hoskins and Simmons 1975). We have initialized the primitive model with the balanced states thus obtained and verified that they are indeed steadystate solutions to the equations of motion.

\section{REFERENCES}

Andrews, D. G., J. R. Holton, and C. B. Leovy, 1987: Middle Atmosphere Dynamics. Academic Press, 489 pp.

Boville, B. A., 1995: Middle atmosphere version of CCM2 (MACCM2): Annual cycle and interannual variability. J. Geophys. Res., 100, 9017-9040.

Butchart, N., S. A. Clough, T. N. Palmer, and P. J. Trevelyan, 1982:
Simulations of an observed stratospheric warming with quasigeostrophic refractive index as a model diagnostic. Quart. J. Roy. Meteor. Soc., 108, 475-502.

Cariolle, D., M. Amodei, M. Deque, J.-F. Mahfouf, P. Simon, and H. Teyssedre, 1993: A quasibiennial oscillation signal in general circulation model simulations. Science, 261, 1313-1316.

Dritschel, D. G., and L. M. Polvani, 1992: The roll-up of vorticity strips on the surface of a sphere. J. Fluid Mech., 234, 47-69.

_ , and R. Saravanan, 1994: Three-dimensional quasigeostrophic contour dynamics, with an application to stratospheric vortex dynamics. Quart. J. Roy. Meteor. Soc., 120, 1267-1298.

Hack, J. J., B. A. Boville, B. P. Briegleb, J. T. Kiehl, P. J. Rasch, and D. L. Williamson, 1993: Description of the NCAR community climate model (CCM2). NCAR Tech. Note NCAR/TN$382+$ STR, 108 pp. [Available from National Center for Atmospheric Research, P.O. Box 3000, Boulder, CO 80307.]

Hamilton, K., R. J. Wilson, J. D. Mahlman, and L. J. Umscheid: 1995: Climatology of the SKYHI troposphere-stratosphere-mesosphere general circulation model. J. Atmos. Sci., 52, 5-43.

Haynes, P. H., 1990: High-resolution three-dimensional modeling of stratospheric flows: quasi-2D turbulence dominated by a single vortex. Topological Fluid Mechanics, H. K. Moffat and A. Tsinober, Eds., Cambridge University Press, 345-354.

— tracers due to large-scale differential advection. J. Atmos. Sci., 54, 1121-1136.

Hoskins, B. J., and A. J. Simmons, 1975: A multi-layer spectral model and the semi-implicit method. Quart. J. Roy. Meteor. Soc., 101, $637-655$.

- M. E. McIntyre, and A. W. Robertson, 1985: On the use and significance of isentropic potential vorticity maps. Quart. J. Roy. Meteor. Soc., 111, 877-946.

Jackson, D. R., and L. J. Gray, 1994: Simulation of the semiannual oscillation of the equatorial middle atmosphere using the extended UGAMP general circulation model. Quart. J. Roy. Meteor. Soc., 120, 1559-1588.

Juckes, M. N., 1989: A shallow water model of the winter stratosphere. J. Atmos. Sci., 46, 2934-2955.

- and M. E. McIntyre, 1987: A high resolution, one-layer model of breaking planetary waves in the winter stratosphere. Nature, 328, 590-596.

Kalnay, E., and Coauthors, 1996: The NCEP/NCAR 40-Year Reanalysis Project. Bull. Amer. Meteor. Soc., 77, 437-471.

Labitzke, K., 1981: The amplification of height wave 1 in January 1979: A characteristic precondition for the major warming in February. Mon. Wea. Rev., 109, 983-989.

Lait, L. R., 1994: An alternative form for potential vorticity. J. Atmos. Sci., 51, 1754-1759.

Leovy, C. B., C. R. Sun, M. H. Hitchman, E. E. Remsberg, J. M. Russell, L. L. Gordley, J. C. Gille, and L. V. Lyjak, 1985: Transport of ozone in the middle atmosphere: Evidence for planetary wave breaking. J. Atmos. Sci., 42, 230-244.

Mariotti, A., B. Legras, and H. Teitelbaum, 1997: Comparison between vertical ozone soundings and reconstructed potential vorticity maps by contour advection with surgery. J. Geophys. Res., 102, 6231-6142.

Matsuno, T., 1970: Vertical propagation of stationary planetary waves in the winter Northern Hemisphere. J. Atmos. Sci., 27, 871-883.

_ 1971: A dynamical model of the stratospheric sudden warming. J. Atmos. Sci., 28, 1479-1494.

McIntyre, M. E., 1982: How well do we understand sudden stratospheric warmings? J. Meteor. Soc. Japan, 60, 37-65.

_ 1991: Atmospheric dynamics: Some fundamentals with observational implications. Proceedings of the International School of Physics "Enrico Fermi," CXV Course, J. C. Gille and G. Visconti, Eds., North-Holland, 313-386.

and T. N. Palmer, 1983: Breaking planetary waves in the stratosphere. Nature, 305, 593-600.

, and - 1984: The "surf zone" in the stratosphere. J. Atmos. Terr. Phys., 46, 825-849. 
Mechoso, C. R., 1990: The final warming of the stratosphere. $D y-$ namics, Transport and Photochemistry in the Middle Atmosphere of the Southern Hemisphere, A. O'Neill, Ed., Kluwer, 55-69.

Norton, W. A., 1994: Breaking Rossby waves in a model stratosphere diagnosed by a vortex-following coordinates and a contour advection technique. J. Atmos. Sci., 51, 654-673.

O'Neill, A., and V. D. Pope, 1988: Simulations of linear and nonlinear disturbances in the stratosphere. Quart. J. Roy. Meteor. Soc., 114, 1063-1110.

Plumb, R. A., 1981: Instability of the distorted polar night vortex: A theory of stratospheric warmings. J. Atmos. Sci., 38, 2514-2531. - and Coauthors, 1994: Intrusions into the lower stratospheric arctic vortex during the winter of 1991/1992. J. Geophys. Res., 99, 1089-1106.

Polvani, L. M., 1992: Two-layer geostrophic vortex dynamics. Part 2. Alignment and two-layer V-states. J. Fluid Mech., 225, 241 270.

_ , and R. A. Plumb, 1992: Rossby wave breaking, microbreaking, filamentation and secondary vortex formation: The dynamics of a perturbed vortex. J. Atmos. Sci., 49, 462-476.

— D. W. Waugh, and R. A. Plumb, 1995: On the subtropical edge of the stratospheric surf zone. J. Atmos. Sci., 52, 1288-1309.

Randel, W. J., 1992: Global atmospheric circulation statistics, 1000$1 \mathrm{mb}$. NCAR Tech. Note NCAR/TN-366+STR, 256 pp. [Available from National Center for Atmospheric Research, P.O. Box 3000, Boulder, CO 80307.]

_ 1993: Ideas flow on the Antarctic vortex. Nature, 364, 105106.

Salby, M. L., R. R. Garcia, D. O’Sullivan, and P. Callaghan, 1990a: The interaction of horizontal eddy transport and thermal drive in the stratosphere. J. Atmos. Sci., 47, 1647-1665.

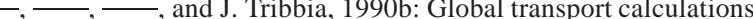
with an equivalent barotropic system. J. Atmos. Sci., 47, 188214.

—, D. O'Sullivan, R. R. Garcia, and P. Callaghan, 1990c: Air motion accompanying the development of a planetary wave critical layer. J. Atmos. Sci., 47, 179-1203.

Schoeberl, M. R., and D. L. Hartman, 1991: The dynamics of the stratospheric polar vortex and its relation to springtime ozone depletions. Science, 251, 46-52.

- and - 1995: A multiple-level trajectory analysis of vortex filaments. J. Geophys. Res., 100, 25 801-25 815.

Simmons, A. J., and R. Strufing, 1981: An energy and angular momentum conserving scheme, hybrid coordinates and mediumrange weather prediction. ECMWF Tech. Rep. 28, 68 pp.

Sutyrin, G. G., J. C. McWilliams, and R. Saravanan, 1998: Corotating stationary states and vertical alignment of geostrophic vortices with thin cores. J. Fluid Mech., 357, 321-349.

Waugh, D. W., 1993: Contour surgery simulations of a forced polar vortex. J. Atmos. Sci., 50, 714-730.

— , and D. G. Dritschel, 1999: Vertical structure of Rossby wave breaking: Dependence on polar vortex vertical structure. J. Atmos. Sci., 56, 2359-2375.

_ polar vortices using elliptical diagnostics. J. Atmos. Sci., 56, $1594-1613$.

- , and Coauthors, 1994: Transport of material out of the stratospheric Arctic vortex by Rossby wave breaking. J. Geophys. Res., 99, 1071-1088.

Yoden, S., and K. Ishioka, 1993: A numerical experiment on the breakdown of a polar vortex due to forced Rossby waves. $J$. Meteor. Soc. Japan, 71, 59-72. 